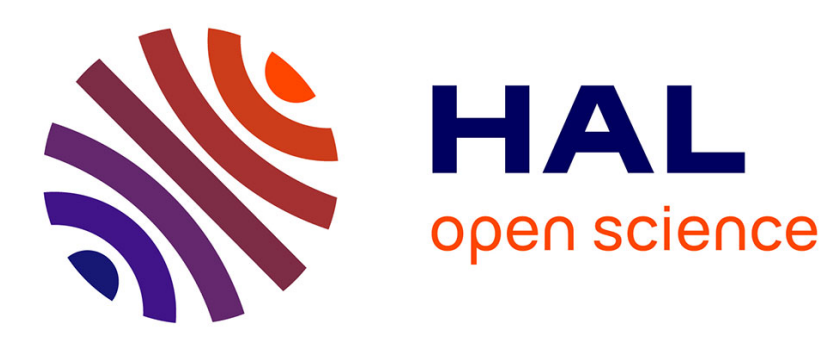

\title{
The key role of massive stars in Oort cloud comet dynamics
}

\author{
M. Fouchard, Ch. Froeschlé, H. Rickman, G.B. Valsecchi
}

\section{To cite this version:}

M. Fouchard, Ch. Froeschlé, H. Rickman, G.B. Valsecchi. The key role of massive stars in Oort cloud comet dynamics. Icarus, 2011, 10.1016/j.icarus.2011.04.012 . hal-00768796

\section{HAL Id: hal-00768796 https://hal.science/hal-00768796}

Submitted on 24 Dec 2012

HAL is a multi-disciplinary open access archive for the deposit and dissemination of scientific research documents, whether they are published or not. The documents may come from teaching and research institutions in France or abroad, or from public or private research centers.
L'archive ouverte pluridisciplinaire HAL, est destinée au dépôt et à la diffusion de documents scientifiques de niveau recherche, publiés ou non, émanant des établissements d'enseignement et de recherche français ou étrangers, des laboratoires publics ou privés. 


\section{Accepted Manuscript}

The key role of massive stars in Oort cloud comet dynamics

M. Fouchard, Ch. Froeschlé, H. Rickman, G.B. Valsecchi

PII:

S0019-1035(11)00147-3

DOI:

10.1016/j.icarus.2011.04.012

Reference:

YICAR 9789

To appear in:

Icarus

Received Date:

15 January 2011

Revised Date:

30 March 2011

Accepted Date:

14 April 2011

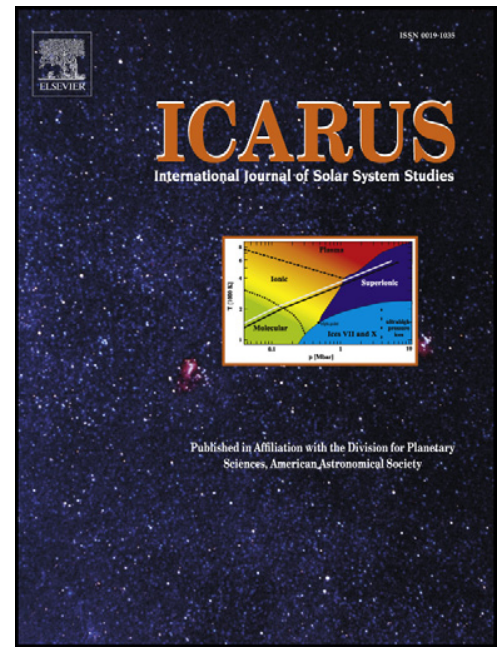

Please cite this article as: Fouchard, M., Froeschlé, Ch., Rickman, H., Valsecchi, G.B., The key role of massive stars in Oort cloud comet dynamics, Icarus (2011), doi: 10.1016/j.icarus.2011.04.012

This is a PDF file of an unedited manuscript that has been accepted for publication. As a service to our customers we are providing this early version of the manuscript. The manuscript will undergo copyediting, typesetting, and review of the resulting proof before it is published in its final form. Please note that during the production process errors may be discovered which could affect the content, and all legal disclaimers that apply to the journal pertain. 


\title{
The key role of massive stars in Oort cloud comet dynamics
}

\author{
M. Fouchard ${ }^{\mathrm{a}, *}$, Ch. Froeschlé ${ }^{\mathrm{b}}$, H. Rickman ${ }^{\mathrm{c}, \mathrm{d}}$, G.B. Valsecchi \\ ${ }^{a}$ LAL-IMCCE, Université de Lille 1, 1 Impasse de l'Observatoire, \\ F-59000 Lille, France \\ ${ }^{b}$ Observatoire de la Côte d'Azur, UMR 6202, Bv. de l'Observatoire, \\ B.P. 4229, F-06304 Nice cedex 4, France \\ ${ }^{c}$ PAS Space Research Center, Bartycka 18A, PL-00-716, Warszawa, Poland \\ ${ }^{d}$ Dept. of Physics 8 Astronomy, Uppsala Univ., Box 516, SE-75120 Uppsala, Sweden \\ ${ }^{e}$ IASF-Roma, INAF, via Fosso del Cavaliere 100, I-00133 Roma, Italy
}

\begin{abstract}
The effects of a sample of 1300 individual stellar encounters spanning a wide range of parameter values (mass, velocity and encounter distance) are investigated.Power law fits for the number of injected comets demonstrate the long range effect of massive stars, whereas light stars affect comets mainly along their tracks. Similarly, we show that the efficiency of a star to fill the phase space region of the Oort cloud where the Galactic tides are able to inject comets into the observable region - the socalled "tidally active zone" (TAZ) - is also strongly dependent on the stellar mass. Power laws similar to those for direct injection are obtained for the efficiency of stars to fill the TAZ. This filling of the tidally active zone is crucial for the long term flux of comets from the Oort cloud. Based on long-term Monte Carlo simulations using a constant Galactic tide and a constant flux of stellar encounters, but neglecting the detailed effects of planetary perturbations, we show that this flux essentially results from a two step mechanism: (i) the stellar injection of comets into the TAZ; and (ii) the tidal injection of TAZ comets into the loss cone. We find that single massive stars are able to induce "comet drizzles" corresponding to an increase of the cometary flux of about 40\% - which may last for more than 100 Myr by filling the TAZ to a higher degree than normal. It appears that the stars involved in this process are the same that cause comet showers.
\end{abstract}

Keywords: Comets, dynamics, origin

\section{Introduction}

Long period comets are believed to come from a nearly spherical reservoir of comets - the so-called Oort cloud (Oort, 1950) - extending over roughly a hundred thousand astronomical units. According to Oort,

\footnotetext{
* Corresponding author

Email addresses: fouchard@imcce.fr (M. Fouchard), froesch@obs-nice.fr (Ch. Froeschlé), hans@fysast.uu.se (H. Rickman), giovanni@iasf-roma.inaf .it (G.B. Valsecchi)
} 
due to perturbations by stars passing in the vicinity of the cloud, the perihelia of some comets in this distant region have been reduced enough for those comets to become observable. In fact, until the 1980's stellar perturbation was the unique mechanism considered to be able to inject comets from the Oort cloud into the inner Solar System (heliocentric distance $r<5 \mathrm{AU}$ ). At that time, the important role of the Galactic tides in making Oort cloud comets observable was established. Beginning with the work of Byl (1983), several studies (see Matese and Whitman, 1992; Wiegert and Tremaine, 1999, and references therein) have shown that the distribution of the observed Galactic latitudes of perihelia of new comets correlates with the action of the disk tide (i.e., the tidal force caused by the variation of the Galactic gravitational potential with distance from the mid-plane). Based on this finding and the realization that, judged as a separate mechanism, the disk tide would be on average more efficient than stellar passages in providing new observable comets, stellar perturbations have later tended to be practically neglected as injection mechanism.

More recently, Matese and Lissauer (2002) studied the evolution of Oort cloud comets over a time scale of $5 \mathrm{Myr}$, suffering simultaneously the perturbations due to the Galactic tides and due to stellar impulses. They found that the sum of the separate injection rates of new comets is significantly larger than the combined rate, and they concluded that over this short time scale, both the Galactic tide and the stars are individually able to fill the loss cone efficiently.

In a previous paper (Rickman et al., 2008) - hereafter RFFV08 - we have also explored the dynamical evolution of Oort cloud comets, but over a time scale of 5 Gyr, including the action of both perturbers, i.e., the stellar perturbations and the Galactic tides, simultaneously. Over the first 5 Myr we confirmed the result found by Matese and Lissauer. But over much longer time scales we found the situation to be reversed, the combined flux being larger than the sum of the separate fluxes. When both perturbers act together, a synergy is generated that significantly increases the flux of comets into the observable region (defined by $r<5 \mathrm{AU}$ ). According to RFFV08, one main reason for this synergy is that passing stars cause a randomization of cometary orbital elements and consequently may send comets into orbits that are linked through the Galactic tides with the observable region.

An important finding was published by Kaib and Quinn (2009), dealing with the role of the giant planets in comet injection from the inner region of the Oort cloud (semi-major axis $\left.a<2 \cdot 10^{4} \mathrm{AU}\right)$. They found that this region contributes to the flux of observable comets by means of the planets perturbing the semi-major axis into $a>2 \cdot 10^{4}$ AU, while the perihelia stay at distances $q \sim 10-15$ AU, after which the Galactic tides may bring the perihelia below the limit of observability.

The main purpose of the present paper is to study the influence of stellar perturbations, according to 
their physical characteristics (stellar mass, velocity and impact parameter), on the efficiency of (i) direct injection of comets into the observable region, and (ii) filling of what we call the tidally active zone (see Sect. 3.3). In both cases special attention will be given to the role of stellar masses. In order to analyze the star-induced dynamics in a comprehensive way, our strategy is to proceed in steps. Therefore, in the present paper we limit ourselves to a simplified model (typical of all earlier works of ours), where the planets are assumed to perturb all comets with $q<15$ AU away from the Oort cloud. A full description of the planetary perturbations, capturing the effects of the Kaib-Quinn mechanism, will be the subject of a forthcoming paper. We caution the reader that the results of the present paper are preliminary as far as the real Solar System is concerned, but they will form a necessary background in order to understand the outcome of the full model.

We begin by a brief description of our dynamical model in Sect. 2. The action of single stars is then studied in Sect. 3. The stellar samples and the initial conditions used for the Oort cloud are described in Sect. 3.1. The amount of direct injections of comets into the observable region, in dependence of the stellar encounter characteristics, is presented in Sect. 3.2. Using the definition of the tidally active zone (TAZ) as a function of the semi-major axis obtained in Sect. 3.3, the efficiency of single stars to fill the TAZ is evaluated in Sect. 3.4.

In Section 4 - like in RFFV08 - we perform three kinds of Monte Carlo simulations of Oort cloud evolution. Each simulation is named after the dynamical model used, i.e., the perturbations considered. Model $\mathrm{G}$ is the simulation for which the Galactic tides provide the unique perturber. In Model S(all) the comets are perturbed only by passing stars whose masses span the whole range from $0.21 M_{\odot}$ to $9 M_{\odot}$, and in model $\mathrm{C}($ all) the two perturbers (tides and stars) act together. In addition, two more sets of simulations are made. Here, rather than considering the whole range of stellar masses, we perform simulations like those of models $\mathrm{S}($ all $)$ and $\mathrm{C}($ all), but considering two different subsamples of stars, one defined by masses $M_{\star}<1.2 M_{\odot}$ yielding models $\mathrm{S}$ (light) and $\mathrm{C}$ (light), and the other containing massive stars with $M_{\star}>1.2 M_{\odot}$ yielding models $\mathrm{S}$ (heavy) and $\mathrm{C}$ (heavy). Discussion and conclusions are given in Sect. 5 .

\section{Dynamical modelling}

The Galactic tides and the stellar perturbations are modelled in the same way as in RFFV08. For the tides this means that we account for the full, non-averaged dynamics including both radial and vertical (disk) terms, using a local disk density of $0.1 M_{\odot}$ pc $^{-3}$ (Holmberg and Flynn, 2000) that characterizes the current solar neighbourhood. Our method of integration is a hybrid approach, described in (Breiter et al., 2007; Fouchard et al., 2007). The 
stellar perturbations are treated by the sequential impulse approximation (Rickman et al., 2005), which builds on the improved impulse approximation by Dybczynski (1994). In the C models we use the tidal integrator, interrupting it at the predefined times of stellar encounters, when the computed heliocentric impulses are added to the cometary velocities. All the comets were integrated in parallel.

For a comparison of the model and parameters used in our simulations with those of earlier Oort cloud simulations, we refer to RFFV08.

\section{Effects of individual stars}

\subsection{Stellar samples and initial conditions}

As in RFFV08, we consider 13 types of stars (mostly represented by MK spectral classes), whose characteristics are listed in Table 1. The basic source for these data is García-Sánchez et al. (2001). For each type, we construct five sequences of 20 stellar encounters, occurring at intervals of 250 Myr during a 5 Gyr interval, the first encounter occurring 100 Myr after the beginning of the simulation.

The stellar velocities and encounter geometries are derived in the same way as in RFFV08. This means that - for any given type of star - we construct the encounter velocity from the data in Table 1 as the vector sum of the solar apex velocity and a randomly chosen, isotropically distributed stellar peculiar velocity. The absolute value of the latter is chosen using a Maxwellian distribution with the listed dispersion, and we use the resulting speed as a weight factor (see RFFV08), since stars contribute to the encounter flux in proportion to their velocities (Heisler et al., 1987). The direction of the encounter velocity is taken at random from an isotropic distribution.

The impact parameter $b_{\odot}$ with respect to the Sun is randomly chosen between $3 \times 10^{3}$ AU and $4 \times 10^{5} \mathrm{AU}$ with a uniform distribution in $\log b_{\odot}$. This choice of distribution makes us sample the whole range of encounter distances more equally than in the case of real stellar encounters, where the closest ones are very rare compared to the distant ones.

These 65 stellar encounter sequences (corresponding to a total of 1300 individual encounters) perturb a cloud of $10^{6}$ fictitious comets having the following statistical characteristics. The initial semi-major axes are taken in the interval: $3 \times 10^{3}<a_{0}<1 \times 10^{5} \mathrm{AU}$ with a flat distribution of orbital energy (i.e., inverse semi-major axis). Thus we sample the whole range of energies equally, whereas in simulations of the real Oort cloud - following (Duncan et al., 1987) - a probability density $\propto a^{-3 / 2}$ is often used, giving more weight to the inner core. Like in the case of stellar impact parameters, since 


\begin{tabular}{|c|ccrr|rr|} 
Type & $\begin{array}{r}\text { Mass } \\
\left(M_{\odot}\right)\end{array}$ & Enc. freq. & $\begin{array}{r}v_{\odot} \\
(\mathrm{km} / \mathrm{s})\end{array}$ & $\begin{array}{r}\sigma_{*} \\
(\mathrm{~km} / \mathrm{s})\end{array}$ & $\begin{array}{r}\langle V\rangle \\
(\mathrm{km} / \mathrm{s})\end{array}$ & $\begin{array}{r}\sigma_{V} \\
(\mathrm{~km} / \mathrm{s})\end{array}$ \\
\hline B0 & 9 & 0.005 & 18.6 & 14.7 & 24.6 & 6.7 \\
A0 & 3.2 & 0.03 & 17.1 & 19.7 & 27.5 & 9.3 \\
A5 & 2.1 & 0.04 & 13.7 & 23.7 & 29.3 & 10.4 \\
F0 & 1.7 & 0.15 & 17.1 & 29.1 & 36.5 & 12.6 \\
F5 & 1.3 & 0.08 & 17.1 & 36.2 & 43.6 & 15.6 \\
G0 & 1.1 & 0.22 & 26.4 & 37.4 & 49.8 & 17.1 \\
G5 & 0.93 & 0.35 & 23.9 & 39.2 & 49.6 & 17.9 \\
K0 & 0.78 & 0.34 & 19.8 & 34.1 & 42.6 & 15.0 \\
K5 & 0.69 & 0.85 & 25.0 & 43.4 & 54.3 & 19.2 \\
M0 & 0.47 & 1.29 & 17.3 & 42.7 & 50.0 & 18.0 \\
M5 & 0.21 & 6.39 & 23.3 & 41.8 & 51.8 & 18.3 \\
wd & 0.9 & 0.72 & 38.3 & 63.4 & 80.2 & 28.2 \\
gi & 4 & 0.06 & 21.0 & 41.0 & 49.7 & 17.5 \\
\hline
\end{tabular}

Table 1: Stellar parameters. The types are mostly MK types for main sequence stars; 'wd' indicates white dwarfs, and 'gi' indicates giant stars. The encounter frequencies are given in number per Myr within 1 pc. The following two columns list the solar apex velocity with respect to the corresponding type, and the spherical Maxwellian dispersion of peculiar velocities. The last two columns give the mean heliocentric encounter velocity and its standard deviation.

the present model is not intended to mimic the real Oort cloud, we prefer to use a uniform sampling.

The initial eccentricities $e_{0}$ are chosen with a density function $\propto e_{0}$ in such a way that the perihelia are outside the planetary system, i.e., $q_{0}>32 \mathrm{AU}$. The angular elements $\omega_{0}, \Omega_{0}$ and $M_{0}$ (we use common notations for the orbital elements, and the angles may be defined with respect to an arbitrary frame of reference), like $\cos i_{0}$, are randomly chosen with flat distributions.

The motion of comets is integrated for a maximum of 5 Gyr, and the integration stops if either a perihelion passage occurs with $q<15 \mathrm{AU}$, or the heliocentric distance increases beyond $r=4 \times 10^{5} \mathrm{AU}$. The comet is then considered as lost from the Oort cloud - either by planetary perturbations (the first case) or by tide-induced escape into interstellar space (the second case). The term injection will be reserved for comets that reach the observable zone at perihelion $(q<5 \mathrm{AU})$.

\subsection{Direct injections}

In this Subsection we will present the results of $\mathrm{S}$ models, neglecting the action of Galactic tides.

Table 2 gives the fractions of stars in three groups, corresponding to different mass ranges, which yield numbers of injected comets in different intervals. We note that even with as many as $10^{6}$ comets in our simulated Oort cloud, the statistical sampling of the number of injected comets is rather poor - especially for the lightest stars, where the number is zero in more than half the cases. More than 20 comets are injected 


\begin{tabular}{|c|ccc|} 
Mass $\left(M_{\odot}\right)$ & 9 & {$[1.3,4]$} & {$[0.2,1.1]$} \\
\hline$n_{\text {inj }}=0$ & 6 & 23.6 & 52.9 \\
$n_{\text {inj }}=1$ & 9 & 8.2 & 9.9 \\
$2 \leq n_{\text {inj }} \leq 5$ & 12 & 11.4 & 10.9 \\
$6 \leq n_{\text {inj }} \leq 20$ & 20 & 10.8 & 13.4 \\
$21 \leq n_{\text {inj }} \leq 200$ & 17 & 25.2 & 12.9 \\
$201 \leq n_{\text {inj }}$ & 36 & 20.8 & 0.1 \\
\hline
\end{tabular}

Table 2: Fraction of stars, given as percent, in three different groups - namely, B0 stars $\left(9 M_{\odot}\right)$, A0 to F5 and red giant stars (1.3 to $\left.4 M_{\odot}\right)$, and all the other stars $\left(0.2\right.$ to $\left.1.1 M_{\odot}\right)$ - yielding numbers of injected comets in different ranges.

by most of the B0 stars (the heaviest) and $46 \%$ of the other heavy stars but only $13 \%$ of the light stars.

Let us base our discussion on the classical impulse approximation (Rickman, 1976), which involves the following assumptions: (i) the star moves with constant velocity with respect to the Sun, and the motion follows a straight line, (ii) in accordance with the high stellar speed, the comet is considered at rest in the heliocentric frame during the stellar passage, and (iii) the stellar effect is computed for a star moving from infinity to infinity. This approximation is useful for discussing and interpreting statistical results, although it is not at all accurate in individual cases for the relatively fastmoving comets of the inner core.

With the above assumptions, the heliocentric impulse imparted to the comet is given by:

$$
\Delta \mathbf{V}=\frac{2 G M_{\star}}{V_{\star}}\left(\frac{\mathbf{b}_{C}}{b_{C}^{2}}-\frac{\mathbf{b}_{\odot}}{b_{\odot}^{2}}\right),
$$

where $M_{\star}$ is the stellar mass, $V_{\star}$ is the stellar velocity, and $\mathbf{b}_{\odot}$ and $\mathbf{b}_{C}$ are the vectors from the Sun and the comet, respectively, to the closest points on the stellar trajectory.

For a close encounter with the comet only, we get approximately:

$$
\Delta \mathbf{V} \propto \frac{M_{\star}}{V_{\star} b_{C}}
$$

while in the case of a distant encounter with both the Sun and the comet, as shown by Rickman (1976), we obtain:

$$
\Delta \mathbf{V} \propto \frac{M_{\star} r}{V_{\star} b_{\odot}^{2}},
$$

where $r$ is the heliocentric distance of the comet.

Now, consider the case of close encounters between the star and the injected comets. Our modelled Oort cloud has a flat distribution of $1 / a$ and thus a distribution like $a^{-2}$ in the semi-major axis.In terms of heliocentric distance, the distribution function is then proportional to $r^{-2}$, and the number density of comets in the cloud $(n)$ varies as $r^{-4}$. We want to find the number of comets that may be injected due to close encounters along the track of the star through the Oort cloud.This will depend on the along-track integral of the number density of comets multiplied by the cross-sectional 
area perpendicular to the track, within which the comets may suffer large enough impulses to inject them.

The requirement is for $|\Delta q|$ to overcome a certain value - typically, the 10 AU needed in order to cross the Jupiter-Saturn barrier (this means the range of perihelion distances within the loss cylinder, where the comets are not observable).Let $G$ be the angular momentum of the cometary orbit. Using the approximate relation $q \propto G^{2}$ for high-eccentricity orbits, we obtain:

$$
|\Delta q| \propto G|\Delta G|
$$

If $v_{t}$ is the size of the transverse velocity component, we have: $G=r v_{t}$. Hence, $|\Delta G|=r\left|\Delta v_{t}\right|$, and we may consider $\left|\Delta v_{t}\right|$ to be some standard fraction of $|\Delta \mathbf{V}|$ for a random orientation of the impulse vector. Taking $|\Delta \mathrm{V}|$ from Eq. (2), we find that in order to have $|\Delta q|>10$ AU, we require $b_{C}<b_{\ell}$ with:

$$
b_{\ell} \propto r \cdot \frac{M_{\star}}{V_{\star}}
$$

Here we consider $G$ to be a constant, given by the pre-injection perihelion distance.

The above-mentioned cross-sectional area $(A)$ is hence:

$$
A \propto r^{2} \frac{M_{\star}^{2}}{V_{\star}^{2}}
$$

and in order to estimate the number $\left(N_{S}\right)$ of injected comets, we have to take the along-track integral through the cloud of $n \cdot A$. The latter product varies as $r^{-2}$, and for a spherical cloud with radius $R_{\mathrm{cl}}$ the result is:

$$
N_{S} \propto \frac{M_{\star}^{2}}{V_{\star}^{2}} \cdot \frac{1}{b_{\odot}} \operatorname{arctg}\left(\frac{R_{\mathrm{cl}}}{b_{\odot}}\right)
$$

This is an approximation that holds for $b_{\odot}<<R_{\mathrm{cl}}$, which is the case of interest for us, and we may use the limiting value of $\operatorname{arctg}\left(R_{\mathrm{cl}} / b_{\odot}\right)=\pi / 2$ as a constant factor.

As a result, in case stars inject comets basically by close encounter perturbations, we expect the injected number to scale with the square of the parameter:

$$
I_{\text {close }}=\frac{M_{\star}}{V_{\star} \sqrt{b_{\odot}}} .
$$

We will look for a correlation between $N_{S}$ and $I_{\text {close }}$ as an indicator of the importance of close star-comet encounters as injection mechanism.

Next, consider the case of distant, tidal encounters described by Eq. (3). In this case the injected comets are not situated near the star track but in a region much closer to the Sun. Let us estimate the size of that region, since this will influence the number of injected comets.If we consider the mean 
transverse velocity change to be proportional to the change of total velocity (see above), we get: $\left|\Delta v_{t}\right| \propto M_{\star} r V_{\star}^{-1} b_{\odot}^{-2}$ from Eq. (3). Thus, according to Eq. (4), we obtain the expected change of perihelion distance as:

$$
|\Delta q| \propto r^{2} \cdot \frac{M_{\star}}{V_{\star} b_{\odot}^{2}}
$$

In order for $|\Delta q|$ to overcome the estimated value of $10 \mathrm{AU}$, we see that $r$ must be larger than some $r_{\min }$, where $r_{\min }^{2} \propto V_{\star} b_{\odot}^{2} / M_{\star}$. This means that the injected comets must be outside a 'forbidden region' around the Sun, whose size decreases with increasing values of the parameter:

$$
I_{\text {dist }}=\frac{M_{\star}}{V_{\star} b_{\odot}^{2}}
$$

Hence we expect $N_{S}$ to correlate positively with $I_{\text {dist }}$ and will look for such a correlation as an indicator of tidal interactions as injection mechanism.

We will refer to $I_{\text {close }}$ and $I_{\text {dist }}$ as "strength parameters" for close and tidal encounters, respectively, and the units to be used are solar masses $\left(M_{\odot}\right)$ for $M_{\star}, 40 \mathrm{~km} / \mathrm{s}$ for $V_{\star}$, and $2 \cdot 10^{4} \mathbf{A U} \simeq 0.1$ pc for $b_{\odot}$. The common factor $S_{\star}=M_{\star} / V_{\star}$ may be regarded as a specifically stellar strength parameter independent of the encounter geometry (Dybczyński, 2002). Within each stellar type of Table 1 some scatter of $S_{\star}$ is caused by the spread in velocities as indicated by $\sigma_{V}$, but if we calculate mean values $\left\langle S_{\star}\right\rangle=M_{\star} /\langle V\rangle$ using the listed mean values $\langle V\rangle$, we find that these vary by a factor $\sim 90$ from B0 stars with $\left\langle S_{\star}\right\rangle \simeq 15$ to M5 stars with $\left\langle S_{\star}\right\rangle \simeq 0.16$ in the above units.

These differences are in reality compensated by the different encounter frequencies - also listed in Table 1. Encounters with M5 stars are more than 1000 times more frequent than those with B0 stars. When discussing our results for $N_{S}$ vs $I_{\text {close }}$ and $I_{\text {dist }}$, it is worth keeping in mind the actually expected minimum encounter distances of each stellar type over a 1 Gyr timescale (taken as representative for the long-term evolution of the Oort cloud). Using the listed encounter frequencies, we may calculate the area that yields $1 / 2$ encounters during 1 Gyr for the type of star in question, and we may take the radius of the corresponding circle for the distance of the expected closest encounter. The result is $\sim 60000 \mathrm{AU}$ for B0 stars and $\sim 2000$ AU for M5 stars.

In Fig. 1 we plot $N_{S}$ as found in our simulations versus the strength parameter of the

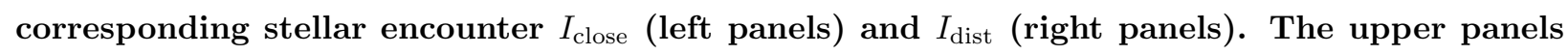
display results for the stellar types with the smallest $\left\langle S_{\star}\right\rangle$. These are K0, K5, wd, M0 and M5 stars. The lower panels show gi, A5, A0 and B0 stars, which have the largest $\left\langle S_{\star}\right\rangle$ values. In 
both cases we use colours to identify the individual types. We also include the intermediate types (F0, F5, G0 and G5) in all diagrams, plotted with grey circles.

The most striking result appears for the high-mass stars. These are seen to show a very good correlation between $N_{S}$ and $I_{\text {dist }}$ over a major part of the plotted range, while no correlation whatsoever is observed for $I_{\text {close }}$. The top, rightmost part of the diagrams exhibit a saturation effect, to which we shall return below. In the lower part of the $I_{\text {dist }}$ diagram, for $N_{S}<10$, we also see an increasing effect of statistical noise. For the low-mass stars the picture is somewhat less clear - partly due to the small values of $N_{S}$ in many cases. However, the correlation of $N_{S}$ with $I_{\text {close }}$ is clearly better than the one with $I_{\text {dist }}$.

If we look at any particular stellar type, the closest encounters with $b_{\odot} \sim 3000-5000$ AU (generally shown by the rightmost points in each diagram) cannot be expected to inject most comets by tidal perturbations. For the high-mass stars we might have seen this as an extra scatter, if there had not been the mentioned saturation effect. But it would not matter in practice, since even over the age of the Solar System not a single such encounter is statistically expected, given the rarity of such stars. For the low-mass stars the correlation with $I_{\text {close }}$ is quite good all the way from the closest encounters to the region, where the statistical noise prevents safe judgements. This means encounter distances up to $b_{\odot} \sim 30000$ AU if not more.

Hence we conclude that as regards stellar injections from the Oort cloud, the stars of highest mass are basically distant, tidal perturbers, while the common, low-mass stars act mostly via close encounters with comets - at least when passing through the inner or central parts of the cloud. We interpret this as a trend with increasing stellar mass for comet injection to move from close encounters along the stellar track to distant ones affecting large parts of the Oort cloud at the same time. Since this trend should be continuous, the stars of intermediate masses (including the solar type stars) are likely to act in both ways, so the mechanism could be different for different, individual comets.

The lines drawn are regression lines, yielding power-law fits for $N_{S}$. In the case of lowmass stars we have excluded all the M5 stars and those other stars that yield $N_{S}<5$ for lack of statistical significance. For the high-mass stars we excluded the saturated region with $I_{\text {dist }}>0.1$. The mathematical expressions for the power laws are:

$$
N_{S}=I_{\text {close }}^{1.82} \times 10^{7.04}
$$

(low-mass stars), and:

$$
N_{S}=I_{\mathrm{dist}}^{0.89} \times 10^{3.96}
$$



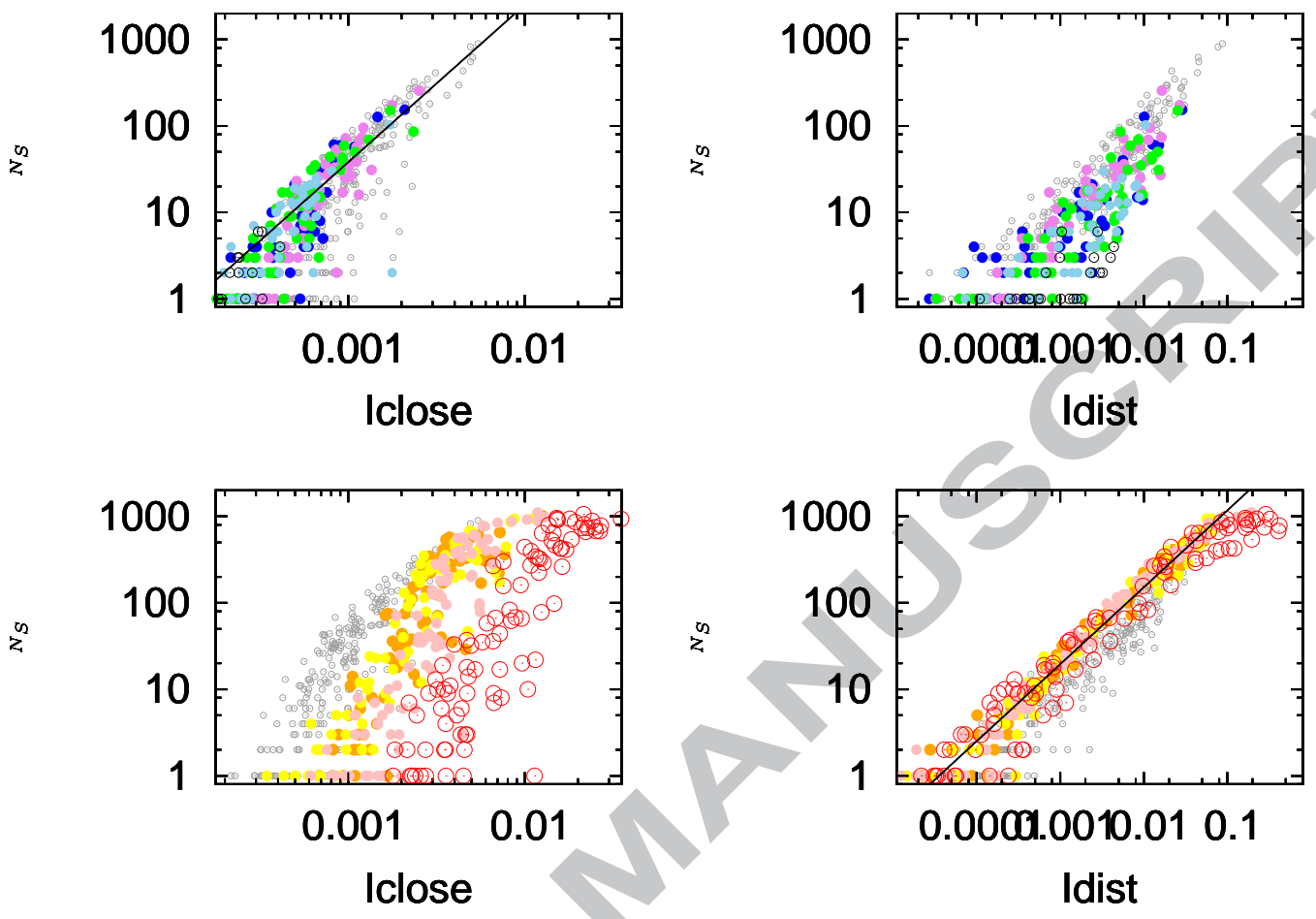

Figure 1: Number of comets entering the observable region due to individual stellar encounters versus $I_{\text {close }}$ (left panels) and $I_{\text {dist }}$ (right panels). In the upper panels we plot low-mass stars $\left(M_{\star} \leq 0.9 M_{\odot}\right)$, using violet colour for K0 stars, blue for K5 stars, green for white dwarf stars, skyblue for M0 stars (in all cases filled circles), and open black circles for M5 stars. In the lower panels we plot high-mass stars $\left(M_{\star}>2 M_{\odot}\right)$, using orange colour for giant stars, yellow for A5 stars, pink for A0 stars (in all cases filled circles), and open red circles for B0 stars. The grey circles indicate the stars of intermediate masses (F0, F5, G0 and G5 stars). We have plotted regression lines (linear fits in log scale) at the upper left for low-mass stars, and at the lower right for high-mass stars.

(high-mass stars). The scaling factors play the role of referring $N_{S}$ to an Oort cloud with $10^{6}$ comets. The power law indices of the above formulae are defined with standard deviations of \pm 0.11 for $I_{\text {close }}$ and \pm 0.01 for $I_{\text {dist }}$. The standard deviations of the exponents entering into the scaling factors are \pm 0.39 and \pm 0.13 , respectively. Note the good agreement of our result for the $I_{\text {close }}$ index with the theoretical value of 2 derived above.

Our results for injection probabilities due to stellar encounters should be compared with those of Dybczyński (2002). We find much larger values than he did, but this is essentially a result of different initial conditions. Instead of choosing the initial cometary orbits with perihelion distances $q>50$ AU (Dybczyński, 2002), we start the simulations with $q>32 \mathrm{AU}$, and each new stellar encounter brings some comets closer to the loss cone so that the next star may act on comets with $q$ closer to 15 AU. Apart from this difference, we note a general similarity of the results - e.g., Dybczyński (2002) found a variation of injection efficiency for 
$b_{\odot}=30000$ AU that is slower for large stellar strength parameters than for small ones (cf. his Fig. 6), and this may be interpreted using our results as a shift from tidal injections in the first case to close encounters in the second.

Our results demonstrate quantitatively that heavy stars are long-range perturbers, typically injecting comets from large parts of the Oort cloud without having to pass very close to the Sun, while low-mass stars in general affect comets mainly near their tracks and need to pass very close to the Sun in order to induce large numbers of injections. In reality, of course, such close encounters are very rare, and even though high-mass stars have much lower encounter frequencies (see Table 1), the latter enjoy the advantage of not having to pass as close in order to cause large effects.

The last feature to be noted in Fig. 1 is an apparent saturation of the injected number at about 1000. While the low-mass stars never reach this level, the high-mass stars sometimes do. The reason for the saturation is the complete filling of the loss cone (cf. RFFV08). This orbital domain, defined by $q<15 \mathrm{AU}$, is normally almost empty in our model of the cloud, and it gets repopulated by each stellar encounter. However, these encounters can do no more than thermalize ${ }^{1}$ the velocity distribution of the comets - there is no reason for them to aim in particular at the loss cone when perturbing the velocities or to overpopulate any part of it, like for instance, the observability zone. Therefore the number of injected comets has a maximum given by the number of comets in our modelled Oort cloud, which would have $q<5$ AU in a thermalized distribution.

This number is easy to estimate, using the fraction $2 q_{o} / a$ with $q_{o}=5$ AU (Bailey and Stagg, 1988) and the probability density function $f(a)$. Integrating the product of these with respect to $a$ over the extent of the cloud, and normalizing to a total population of $10^{6}$ comets, we get a value between 1000 and 2000, in agreement with the ceiling observed in Fig. 1.

From these results, even though the distribution of encounter distances that we use is very different from reality, our results suggest high-mass stars to be at least as important as lowmass stars for direct injection of comets from the Oort Cloud in the long run despite their much lower encounter frequency. This will be further discussed in Sect. 4.

\subsection{The tidally active zone}

The synergy observed in RFFV08 during periods outside comet showers may result largely from the fact that stellar encounters randomize the comet orbital elements and consequently repopulate the regions of

\footnotetext{
${ }^{1}$ The fact that we exclude comets with $q<32$ AU makes very little difference and the effect may be neglected.
} 
phase space, from which the tides may inject the comets into the observable region.

Let us consider the case of integrable dynamics involving only the Galactic disk tide. In this framework, we have to assume that the semi-major axes of the comets are small enough to allow the averaging of the equations of motion with respect to the mean anomaly (see Heisler and Tremaine, 1986; Breiter et al., 1996; Fouchard et al., 2005), and we neglect the radial component of the tides as well as stellar encounters.

In this case it is well known (Breiter et al., 1996) that the evolution of the perihelion distance is strictly periodic. Hence, a comet can be injected into the observable region only, if the minimum value of the perihelion distance over one cycle is smaller than 5 AU. The phase space region of the Oort cloud which contains all such comets will be called the tidally active zone (TAZ).

According to the work of Matese and Whitman (1992) one can easily define the TAZ, using the integrability of the system. Indeed, the minimum value of the angular momentum $G_{\min }$ of a comet orbit is given by:

$$
\begin{aligned}
G_{\min }^{2}= & \frac{1}{8}\left[5 L^{2}+5 H^{2}-G_{0}^{2}-\right. \\
& \left.\sqrt{\left(5 L^{2}+5 H^{2}-G_{0}^{2}\right)^{2}-80 L^{2} H^{2}}\right]
\end{aligned}
$$

where:

$$
G_{0}^{2}=H^{2}+\left(1-\frac{H^{2}}{G^{2}}\right)\left(G^{2}+5\left(L^{2}-G^{2}\right) \sin ^{2} \omega\right),
$$

and we have $L=\sqrt{\mu a}, G=\sqrt{\mu a\left(1-e^{2}\right)}$ and $H=\sqrt{\mu a\left(1-e^{2}\right)} \cos i$.

Consequently, under an integrable Galactic tide, the minimum value that the perihelion distance of a comet can reach is:

$$
q_{\min }=a\left(1-\sqrt{1-\frac{G_{\min }^{2}}{\mu a}}\right) .
$$

The TAZ can then be obtained as the region of phase space in which, according to the equations above, $L, G$ and $H$ are such that $q_{\min }<5$ AU.We may compare with the work of Maciejewski and Prȩtka (1998), who gave a criterion for an Oort cloud comet in order certainly not to become observable under the effects of a quasi-integrable tide. Specifically, considering only the normal component of the tide but without averaging, they computed a global minimum $r_{\min }$ of the heliocentric distance depending only on the total energy and the normal component of the angular momentum. Doing this for a comet on a given orbit, in case the result is $r_{\min }>5 \mathrm{AU}$, the comet would never become observable according to our criterion. However, because the minimum is global, $r_{\min }$ is not the same as the minimum heliocentric distance reached by 
this comet with its specific values of initial eccentricity and argument of perihelion during an arbitrarily long time. The latter is instead approximated by our result for $q_{\min }$. This conclusion is independent of the fact that their system was not averaged.

Consequently, their criterion might be efficient for speeding up simulations, since one would integrate only the comets for which $r_{\min }<5$ AU (as far as neither the radial component of the tide nor stars are taken into account). Because $5 \mathrm{AU} \geq q_{\min } \geq r_{\min }$ for our TAZ, all its comets would be integrated using the (Maciejewski and Prȩtka , 1998) criterion, but if we would define the TAZ using $r_{\min }$ instead of $q_{\min }$, we would have many comets inside that TAZ, which in fact cannot enter the observable region. Consequently, even though we have one additional hypothesis since we have averaged our equations of motion, we will use the above $q_{\min }$ to define the TAZ.

Let us now consider three regions: the inner region $(a<20000 \mathrm{AU})$, the central one $(20000<a<$ $50000 \mathrm{AU})$, and the outer one $(50000<a<100000 \mathrm{AU})$. The part of the cloud outside these regions is not considered, since it is empty at the beginning and remains almost empty during the integrations. Furthermore, we cannot consider an integrable tide for such comets. Strictly speaking, integrability only holds true for the inner region and part of the central region.

Figure 2 shows the fraction of comets in the TAZ (in percent) versus time, as found in the Model G simulation, for the three regions of the Oort cloud and for the entire Oort cloud (excluding any comets with $a>100000 \mathrm{AU})$. Note that this tide model includes the radial component in addition to the obvious fact that we treat non-averaged equations of motion. We may thus expect important effects (see below) for the outer Oort cloud region, when applying the theoretical TAZ to the simulation results. The three regions are plotted using different colours, while the black curve shows the result for the entire cloud.

In the beginning of the integration, i.e., at time $t=0$, the filling of the TAZ is generally at its maximum. The outer region shows a special behaviour, as discussed below. Moreover, since our choice of initial orbital elements assumes that the Oort cloud is thermalized, we may consider that the initial fraction corresponds to a completely filled TAZ. The fractions of comets in the TAZ when it is completely filled are thus: $p_{\text {full }}=5.85 \%, 2.63 \%$ and $1.80 \%$ for the inner, central and outer regions of the cloud, respectively. For the entire Oort cloud we have $p_{\text {full }}=5.42 \%$. We note that $p_{\text {full }}$ is higher for the regions closer to the Sun. This can be realized from Eq. (13), since it is easily seen that $G_{\min }^{2} \propto \mu a$, and thus $q_{\min } \propto a$. Hence, everything else being fixed, $q_{\min }$ decreases for decreasing semi-major axis, and consequently, the relative measure of the TAZ increases. 


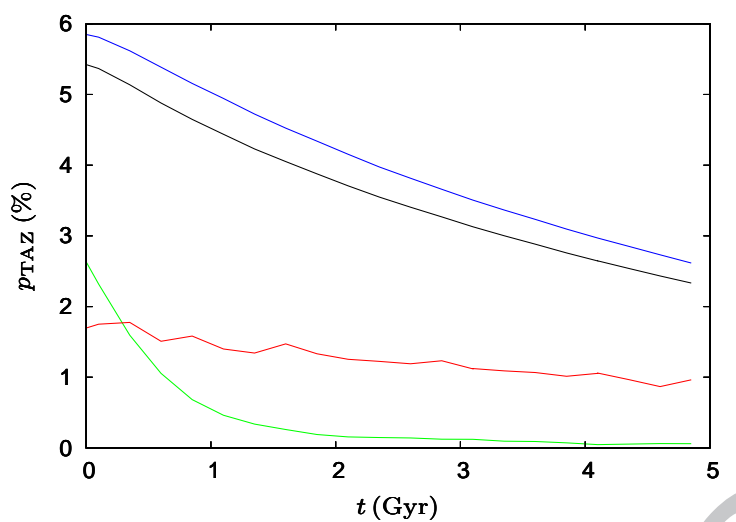

Figure 2: Percentage of comets in the TAZ for different regions of the Oort cloud. The blue, green, red and black curves illustrate, respectively, the inner, central, outer, and entire Oort cloud.

As time goes on, the TAZ gets depleted, since the comets that enter the observable region on account of $q<5 \mathrm{AU}$ get removed from the integration. Let us now consider this depletion of the TAZ in more detail for each region of the Oort cloud separately.

- In our dynamical model, no comets of the inner region are able to jump the Jupiter-Saturn barrier, since the tides are not strong enough to decrease the perihelion distance from $q \geq 15$ AU to below $5 \mathrm{AU}$ in only one orbital period. Hence, even though the TAZ gets depleted as seen in the Figure, there are no comets injected into observable orbits.

The relatively slow depletion of the TAZ is explained as follows. As specified in Sect. 3.1, the simulation is stopped and the comet is lost as soon as its heliocentric distance $r \leq r_{c}=15 \mathrm{AU}^{2}$. As time goes on, the TAZ population is reduced in relative terms, since more comets located in the TAZ reach the limiting value $r_{c}=15 \mathrm{AU}$ than comets that are outside the TAZ. The low speed of the depletion is due to the very long periods of the perihelion cycle for inner region comets. Indeed, this period is proportional to $1 / P_{\text {orb }}$, where $P_{\text {orb }}$ is the orbital period (see Matese and Whitman, 1992; Breiter et al., 1996).

- The depletion of the TAZ is particularly evident for the central region (see Fig. 2) and occurs mainly during the first billion years. This fast depletion is explained by the fact that the period of the perihelion cycle $\left(P_{q}\right)$ is shorter than for the inner region. In fact, most comets in the inner region have $P_{q}>5 \mathrm{Gyr}$, so there is not enough time in our simulation for the TAZ to get fully depleted. But in the central region we can expect that most comets have $P_{q}<5$ Gyr, so the TAZ should have time

\footnotetext{
${ }^{2}$ If the perihelion distance at this time is $q<5 \mathrm{AU}$, we record a case of comet injection.
} 
to get nearly fully depleted. However, we see that even at the end of the integration, the TAZ is not entirely empty.

This fact is explained by two properties of the dynamics. First, we recall that the integration of a comet is stopped, when its heliocentric distance becomes less than 15 AU. Thus, a necessary condition for a TAZ comet to be excluded is that at the time when its perihelion distance is smaller than $15 \mathrm{AU}$, the comet should actually be close to its perihelion. For large semi-major axes, the probability of this event decreases as the inverse of the square of the orbital period (Fouchard et al., 2010). A comet may hence stay in the TAZ even if the period of its perihelion cycle is short (implying a large orbital period).

Second, and probably the main point, the real dynamics is not integrable, since the assumption of an orbit-averaged Hamiltonian as implicit in our definition of the TAZ breaks down. In addition there is also the radial component of the tide, which is not considered in the TAZ definition. As a result, the TAZ border cannot be strictly hermetic. This permeability of the TAZ increases with the semi-major axis, since the influence of the non-integrable part of the dynamics (including the radial component) increases with increasing semi-major axis (Heisler and Tremaine, 1986; Fouchard et al., 2005).

- These two properties obviously apply to the outer region as well. The permeability of the TAZ is now very high. This is why the filling of the TAZ is almost constant with time for this region of the Oort cloud.

\subsection{Filling of the tidally active zone by stars}

What happens when the stars are at work? Each time a star passes through the Oort cloud, it perturbs the orbital elements of many comets - notably those placed near its track - injecting some of them into the TAZ, and ejecting some of them from the TAZ. If the Galactic tides are not at work, from a statistical point of view, nothing happens. But, if the tidal effects are included, the density inside the TAZ will be lower than the density outside it. Then the action of the stars will be to fill the TAZ, thereby tending to balance these two densities. We will now study this phenomenon by simulating the evolution of an Oort cloud in the presence of both the Galactic tides and the stellar encounter sequences described in Sect. 3.1.

In order to quantify the effect of a star, we define a TAZ filling efficiency at time $t^{\star}$ (the time of perihelion passage of the star, when its perturbations on the comets are applied) by:

$$
f=\frac{n_{\mathrm{inj}}\left(t^{\star}\right)}{n_{\text {Oort }}} \cdot \frac{1}{p_{\text {full }}},
$$


where $n_{\mathrm{inj}}$ is the number of comets passing from outside the TAZ at $t^{\star}-\epsilon$ to inside the TAZ at $t^{\star}+\epsilon ; \epsilon$ is a short interval of time, $n_{\text {Oort }}$ is the number of comets at $t^{\star}+\epsilon$ in the Oort cloud, and $p_{\text {full }}$ is the full TAZ fraction of the Oort cloud as given in Sect. 3.3. Obviously, one may compute $f$ for each sub-region of the Oort cloud (inner, central, or outer). The quantity $\epsilon$ should be taken sufficiently small in order that no, or at most very few, comets are removed from the integrations between $t^{\star}-\epsilon$ and $t^{\star}+\epsilon$. We have taken $\epsilon=1000 \mathrm{yr}$.

Figure 3 shows the value of $f$ in percent versus the impact parameter with respect to the Sun $\left(b_{\odot}\right)$ for each cloud region and for all the stars used in our simulations. The following observations may be made.

- All plots show a rather clear separation of the efficiency factor with respect to the stellar type, which shows that the stellar mass and velocity are key parameters for TAZ filling. A saturation effect is often seen, whereby - for obvious reasons - the $f$ values are limited to $\simeq 100 \%$ or less.

- As regards the inner Oort cloud region, (1) for low-mass stars (wd stars and lighter), $f$ is at most $\mathbf{2 4 \%}$ for a white dwarf with a solar impact parameter $b_{\odot} \sim 3300$ AU. For $b_{\odot}>$ 12000 AU, $f$ is always smaller than $\mathbf{3 . 5 \%}$ and drops to values below $\mathbf{1 \%}$ for $b_{\odot}>25000 \mathrm{AU}$.

(2) For the massive B0 stars, values of $f>80 \%$ are reached, when the impact parameters $b_{\odot}$ are less than 5000 AU, while for $b_{\odot}>25000$ AU, $f$ decreases below $\mathbf{1 5 \%}$.

- For the central Oort cloud region, the picture changes drastically. High-mass stars (A5 stars and heavier) excluding the B0 type are now able to fill up $70 \%$ of the TAZ for impact parameters around 20000 AU or less. For impact parameters around 50000 AU, up to about $25 \%$ of the TAZ is refilled by these stars (and up to 50\% for B0 stars). Regarding the low-mass stars, the best filling efficiencies are $60-70 \%$ for impact parameters less than 4000 AU. For impact parameters around $10000 \mathrm{AU}$ and $50000 \mathrm{AU}, f$ drops to values around $30 \%$ and $\mathbf{2 . 5 \%}$, respectively, i.e., one order of magnitude smaller than the values obtained for high-mass stars.

- Concerning the outer Oort cloud region, despite the fact that the TAZ is not well defined as already pointed out in Sect. 3.3, we still observe that massive stars are 5-10 times more efficient than light stars in filling the TAZ. The filling efficiency can reach high values even for large impact parameters (it may be larger than $100 \%$ at $d_{\odot}>60000$ AU for B0 stars). However, one should note that even the tide alone is able to fill the TAZ in this case. Indeed, it appears that during the time span $\Delta t \simeq 250 \mathrm{Myr}$ 

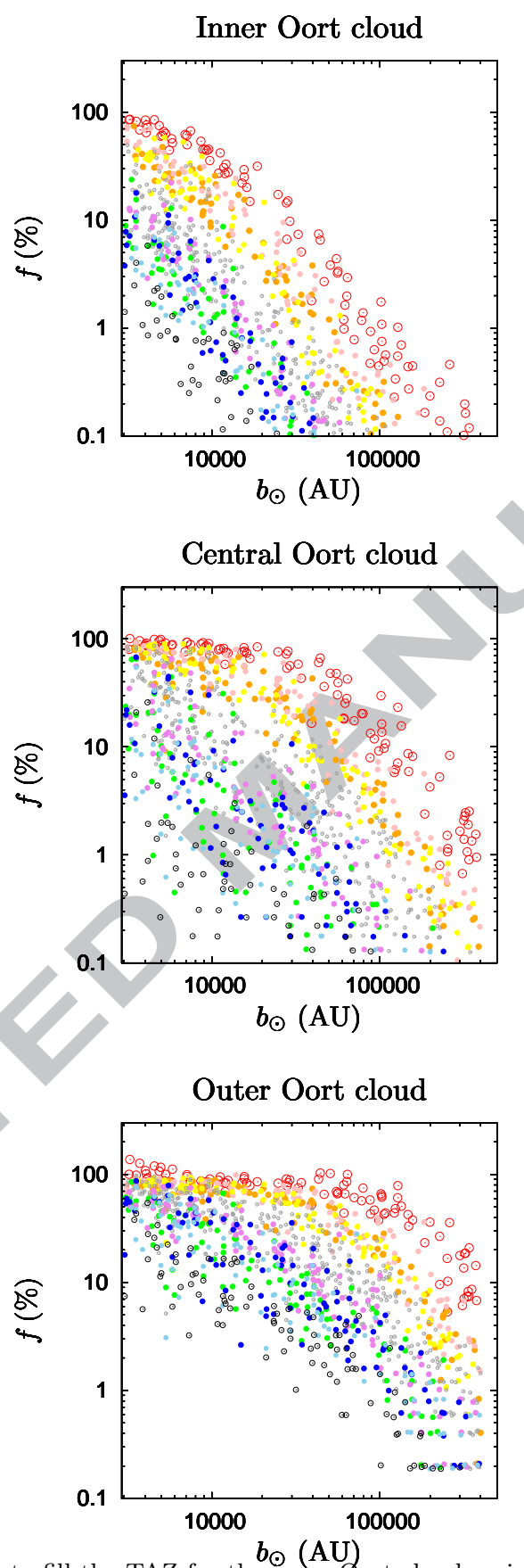

Figure 3: Efficiency $f$ of individual stars to fill the TAZ for the inner Oort cloud region (top diagram), central region (middle diagram) and outer region (bottom diagram) versus the solar impact parameter. The colour codings of stellar types are the same as in Fig. 1.

separating the stellar encounters of our simulations, the tides fill the outer TAZ almost completely. This demonstrates the large permeability of the TAZ at such large semi-major axes.

In Fig. 4 we plot the value of the TAZ filling efficiency $f$ as computed between the stellar encounters 


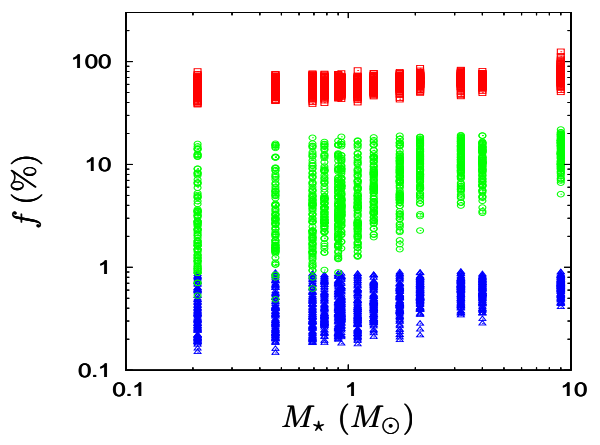

Figure 4: For the inner (blue open triangles), central (green open circles) and outer Oort cloud (red open squares), we plot the efficiency $f$ of the tides to fill the TAZ between stellar encounters, versus the stellar masses.

versus the stellar mass of the preceding encounter, for the three regions of the Oort cloud. Specifically, we count $n_{\text {inj }}$ from $t^{\star}+\epsilon$ for one stellar encounter to $t^{\star}-\epsilon$ for the next one, and we use $n_{\text {Oort }}$ at the latter occasion. In this case the TAZ filling is only due to the tides. We see that for the three regions the values of $f$ are gathered in different ranges: $[0.15 \%, 0.88 \%],[0.74 \%, 21.2 \%]$ and $[38 \%,>100 \%]$ for the inner, central and outer Oort cloud, respectively. This is a demonstration that the TAZ is indeed almost hermetic in the inner Oort cloud, moderately permeable for the central Oort cloud and completely permeable for the outer Oort cloud.

Interestingly, we observe a slight dependence on the stellar mass with higher values of $f$ for higher stellar masses. A priori, the stellar perturbations should not affect the TAZ filling, since $f$ is computed between these encounters. Our explanation is that just outside the frontier between the TAZ and the rest of the phase space there is a transition region in which the tide is inefficient (as anywhere outside the TAZ), but the perturbation needed to transfer the comet from this transition region into the TAZ is so small that it can be provided by the non-integrable component of the tide. When the TAZ gets depleted due to comets falling into the loss cone, this mobility acts to resupply comets into the TAZ, whereby the transition region also gets depleted. Since massive stars are more efficient to fill the TAZ, they are also more efficient to fill the transition region, and hence the tides are more efficient to fill the TAZ after such stellar encounters.

Because the inner Oort cloud does not contribute to the flux of observable comets without the direct help of a stellar perturbation ${ }^{3}$, and since the TAZ of the outer Oort cloud is easily refilled by the tides only, we will now concentrate on the central part of the Oort cloud.

For the central Oort cloud, Fig. 5 gives $f$ (in percent) versus $I_{\text {close }}$ (upper panel) and versus $I_{\text {dist }}$ (lower panel) for individual stars. Linear fits in log scale (shown by the straight lines) are

\footnotetext{
${ }^{3}$ Note that this may not be true in reality but is implied by our simplified treatment of planetary perturbations.
} 
given by:

$$
f \propto I_{\text {close }}^{2.74 \pm 0.08}
$$

where the fit has been made for the ensemble of wd, K0, K5 and M0 stars (upper panel), and:

$$
f \propto I_{\text {dist }}^{1.00 \pm 0.02}
$$

for gi, A5, A0 and B0 stars together (lower panel). Note that a cut-off has been made for $I_{\text {dist }}>0.002$ in this case in order to avoid the saturated region.

Comparing Eqs. (14) and (15) with Eqs. (11) and (12), we note that the power law indices are similar. This is an indication that the relative efficiencies of stars to inject comets into the TAZ are similar to those of injecting comets directly into observable orbits.
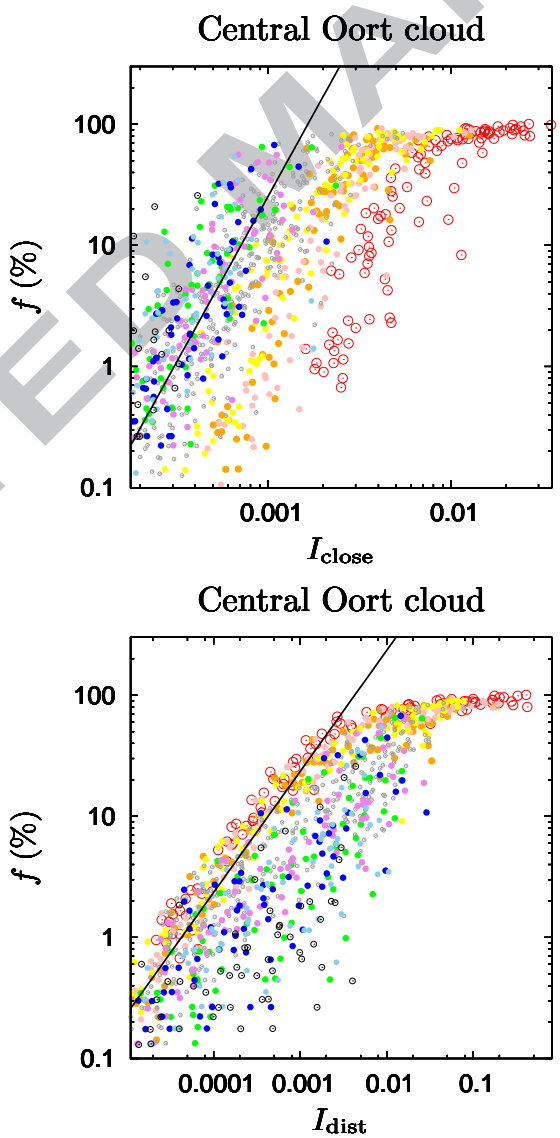

Figure 5: Efficiency $f$ of individual stars to fill the TAZ of the central Oort cloud versus $I_{\text {close }}$ (upper panel) and $I_{\text {dist }}$ (lower panel). The colour coding of the points is the same as in Fig. 3. The full-drawn lines indicate linear fits in log scale. 


\subsection{Long term dynamics}

Let us now study the effect of the TAZ filling efficiency on the flux of observable comets over a long time scale. We will consider two sets of stars, one including 20 A5 stars $\left(M_{\star}=2.1 M_{\odot}\right)$ and the other including $20 \mathrm{~K} 0$ stars $\left(M_{\star}=0.78 M_{\odot}\right)$. The geometries of the encounters are different in the two cases except in the very beginning of the sequence.

We perform three computations including: (i) the Galactic tides only, (ii) stars only, and (iii) both Galactic tides and stars, and we compare the results as in RFFV08. Our model parameters for the cloud are the same as described in Sect. 3.1, i.e., all regions (inner, central and outer) are included. The results of the simulations in terms of numbers of injected comets during 20-Myr intervals over a total time-scale of 5 Gyr are plotted in Fig. 6.

The direct injections (Sect. 3.2) are limited to the time bin of the respective encounter, as shown by the grey spikes. We notice that for similar solar impact parameters, the height of these spikes is always smaller for K0 stars than for the higher-mass A5 stars. This is in agreement with the results found in Sect. 3.2.

We also notice that the combined models (white histograms) show a continuous excess of injected comets with respect to the tide-only models (black histograms) of the kind seen in RFFV08. This reflects the synergy discussed in RFFV08 and is largely explained by the filling of the TAZ induced by stellar perturbations. The lower plots of Fig. 6 show the time evolution of the amount of TAZ filling given by

$$
p_{\mathrm{TAZ}}=\frac{n_{\mathrm{TAZ}}}{n_{\mathrm{CR}}} \cdot \frac{1}{p_{\mathrm{full}}}
$$

in the central Oort cloud region, for the combined model with A5 stars (lower plot of Fig. 6a), and for the combined model with K0 stars (lower plot of Fig. 6b).

Concentrating on times larger than 1 Gyr, and comparing the two kinds of stars, we clearly see a correlation between the filling of the TAZ and the excess of injected comets of the combined models. We may also recognize that the excess jumps to higher levels in connection with the closest, most effective stellar encounters. These results prove that the filling of the TAZ by passing stars is the fundamental mechanism behind the synergy between the Galactic tides and stellar perturbations. As seen from the diagrams, this synergy is able to trigger "comet drizzles" due to single encounters filling the TAZ, inducing a significant increase of the flux of observable comets lasting up to a few hundred Myr.

\section{Cumulative stellar effects in long term simulations}

\subsection{Initial conditions and simulations}

We now consider the same thermalized initial Oort cloud as in RFFV08. Specifically, the cloud is constructed in the same way as in Sect. 3.1, but the semi-major axes are chosen with a probability density $\propto a_{o}^{-1.5}$ (Duncan et al., 1987). 
(a)

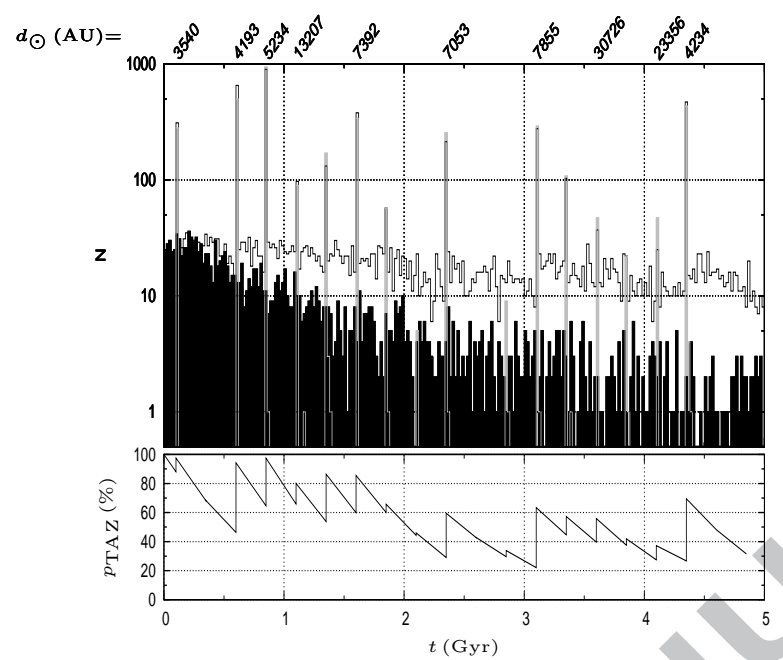

(b)

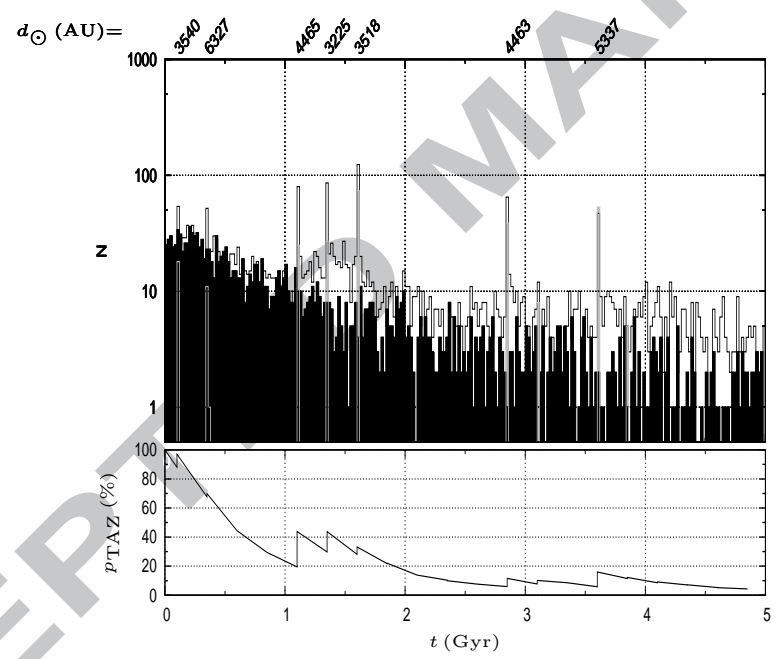

Figure 6: The upper panels show the number of injected comets per $20 \mathrm{Myr}$ versus time. The black histograms correspond to the tide-only model, the grey ones to the star-only model, and the white histograms to the combined model. The impact parameters of the closest encounters are written above each plot. Lower panels: amount of the TAZ filling versus time for the central cloud region. Figure $(a)$ is for A5 stars $\left(2.1 M_{\odot}\right)$ and $(b)$ for K0 stars $\left(0.78 M_{\odot}\right)$.

We also use the same sample of passing stars as in RFFV08, defined as follows: we build a predefined set of 197906 stellar encounters, occurring at random times during an interval of time from $t=0$ to 5 Gyr, with random solar impact parameters up to $d_{\max }=4 \times 10^{5} \mathrm{AU}$, and with random stellar masses and velocities (see Table 1) (Rickman et al., 2004, 2008).

As seen from Table 1, the encounter frequency is much smaller for heavy stars than for light ones, and consequently the number of encounters is much larger for light stars than for heavy stars. In fact, out of a total number of 197906 stellar encounters, 191036 are due to light stars and only 6870 are due to heavy 


\begin{tabular}{|c|ccccc|} 
Model & {$[0-1]$ Gyr } & {$[1-2]$ Gyr } & {$[2-3]$ Gyr } & {$[3-4]$ Gyr } & {$[4-5]$ Gyr } \\
\hline G & 2117 & 801 & 473 & 307 & 252 \\
\hline S (all) & 1367 & 1620 & 1101 & 688 & 480 \\
C (all) & 3703 & 3244 & 2450 & 1782 & 1276 \\
$\langle\tau\rangle$ & $6.29 \%$ & $33.99 \%$ & $55.65 \%$ & $79.1 \%$ & $74.32 \%$ \\
\hline S (heavy) & 855 & 985 & 352 & 306 & 205 \\
C (heavy) & 3075 & 2391 & 1583 & 1259 & 934 \\
$\langle\tau\rangle$ & $3.47 \%$ & $33.87 \%$ & $91.88 \%$ & $105.38 \%$ & $104.38 \%$ \\
\hline S (light) & 810 & 901 & 915 & 622 & 482 \\
C (light) & 3174 & 2617 & 2292 & 1831 & 1487 \\
$\langle\tau\rangle$ & $8.44 \%$ & $53.76 \%$ & $65.13 \%$ & $97.1 \%$ & $102.59 \%$ \\
\hline
\end{tabular}

Table 3: Number of comets entering the observable region during periods of 1 Gyr. Model $\mathrm{G}$ corresponds to the Galactic tide alone, $\mathrm{S}$ to passing stars alone, and $\mathrm{C}$ to Galactic tide and passing stars together (all, heavy and light mean that all, only the heavy or only the light stars are taken into account in the simulations). $\langle\tau\rangle$ is the increment from the sum of the two first rows (Galactic tide plus passing stars separately) to the third row (Galactic tide and passing stars together).

stars.

We recall that for models $\mathrm{S}\left(\right.$ light) and $\mathrm{C}$ (light) only the light stars $\left(M_{\star}<1.2 M_{\odot}\right)$ are taken into account, and for models $\mathrm{S}$ (heavy) and $\mathrm{C}$ (heavy) only the heavy stars $\left(M_{\star}>1.2 M_{\odot}\right)$ are at work.

\subsection{Synergy induced according to the star samples}

In Table 3 we list the number of comets entering the observable region during intervals of 1 Gyr, for the different sets of models described at the end of the Introduction.

Moreover, as in RFFV08, in order to quantify the synergy we have computed the quantities: $\Delta N_{C}=$ $N_{C}-N_{G}-N_{S}$ and $\tau=\Delta N_{C} /\left(N_{S}+N_{G}\right)^{4}$.

Looking at Table 3, we notice that we always have $N_{S}$ (all) $<N_{S}$ (heavy) $+N_{S}$ (light). This means that the intersection between the sets of comets injected by heavy stars and by light stars is not empty. Specifically, we observe from Table 3 that the sum of the injected numbers in the $\mathrm{S}$ (heavy) and $\mathrm{S}$ (light) models exceeds $N_{S}$ (all) by about $20 \%$.

Now, consider the total number of comets injected during the whole time span of 5 Gyr in the S(light) or S(heavy) model, and let us define an efficiency factor of a star sample to inject comets by: $\eta_{S}=($ number of comets injected)/(number of stellar encounters). We get, respectively: $\eta_{\text {light }} \simeq 1.9 \times 10^{-2}$ and $\eta_{\text {heavy }}=$ $39 \times 10^{-2}$.

However, due to the high encounter frequency of light stars (one every $26000 \mathrm{yr}$ ), we have to expect that the stars of the S(light) model will as a rule interfere with each other, as comets are being injected. The number of injected comets may be affected by these interferences, and thus it is not directly comparable to

\footnotetext{
${ }^{4}$ For the all-stars models, a small discrepancy with the results of RFFV08 may be noticed. This is due to a correction of our code in the check of perihelion passages. However, the discrepancy does not change in any sense the results of RFFV08.
} 
the injected number in the S(heavy) model, where the amount of interference is much smaller (one star every $730000 \mathrm{yr})$. Thus, in order to compute $\eta_{\text {heavy }}$ for an encounter frequency similar to the one of light stars, we rather consider the difference between the number of comets injected by all the stars in model $\mathrm{S}($ all) and those injected by the light stars only in model $\mathrm{S}$ (light), i.e., $5256-3730=1526$. We consider the injection of this number of comets to be due to the heavy star encounters. Finally, we compute $\eta_{\text {heavy }}=1526 / 6870$, obtaining: $\eta_{\text {heavy }} \simeq 22 \times 10^{-2}$.

Consequently, even in a full simulation where several stars almost always act simultaneously, heavy stars are at least 10 times more efficient than light stars in order to directly inject comets into the observable region.

As regards the synergy, we note that the values of $\langle\tau\rangle$ after more than 2 Gyr are higher for those combined models where only one subset of stars is included. The main circumstance contributing to this feature is the lower number of injected comets for the star-only models when only one subset of stars is considered. The corresponding number in the combined models is smaller too but by far not to the same extent.

In view of the small number of heavy stars, the high values of $\langle\tau\rangle$ for the heavy-star combined model are striking. As already noted in RFFV08, and as shown in Sect. 3.3, this synergy is mainly explained by the injection of comets by stars into the TAZ.

In Fig. 7 we plot the results of three sets of simulations: those obtained with all (heavy + light) stars (Fig. 7a), with only heavy stars (Fig. 7b), and with light stars only (Fig. 7c). The upper panel of each figure shows the number of comets injected into the observable region (perihelion passages with $q<5$ AU) per period of 20 Myr versus time for three models. The black histograms correspond to the tide-only model (model G), the grey histograms correspond to the star-only models, i.e., models $\mathrm{S}($ all), $\mathrm{S}$ (heavy) and $\mathrm{S}($ light), and the white histograms correspond to the combined models: $\mathrm{C}($ all), $\mathrm{C}$ (heavy) and C(light). Let $N_{G}, N_{S}$ and $N_{C}$ be the numbers of comets plotted in the black, grey and white histograms, respectively. Then the lower panels show the quantity: $\Delta N_{C}=N_{C}-N_{G}-N_{S}$, which measures the synergy arising, when both the Galactic tide and the stars act simultaneously. Figure 7a shows the same results as Fig. 2 of RFFV08.

In RFFV08, we found over the first 500 Myr that the synergy was negative, i.e., the sum of the separate fluxes was larger than the combined flux. As explained by Matese and Lissauer (2002), this result is a typical outcome, when both tides and stars are individually able to fill the loss cone. The same phenomenon is observed in the present results for all three sets of simulations. But, in addition, in the lower panels of Fig. 7 - showing the values of $\Delta N_{C}$ computed every $20 \mathrm{Myr}$ - we see that over the entire 5 Gyr of simulation, 
the synergy parameter $\Delta N_{C}$ becomes negative several times later on, namely, when showers occur. During those periods both the tides and the stars are indeed able to fill the loss cone individually. This phenomenon was not observed in RFFV08, since $\Delta N_{C}$ was then computed over periods of 50 Myr, which smoothed out the effect of the showers.

As may be noticed in Fig. 7b, the flux of comets generated by the heavy star model shows large fluctuations and drops to zero several times. This indicates that the flux is characterized by comet showers completely filling the loss cone, which are separated by quiescent periods, during which the loss cone may become empty. On the contrary, the flux induced by the light stars is rather characterized by a permanently non-empty loss cone. Because strong comet showers occur during the two first billion years for the heavy star model, the two fluxes (heavy stars and light stars) are overall comparable. Note that the relative difference between these two fluxes is much less than the corresponding difference in the number of stars (about a factor 28).

\subsection{TAZ filling and flux of observable comets}

In order to highlight the fundamental role of the TAZ filling to explain the flux of injected comets, we have devised the following experiment. We perform three different Oort cloud simulations (using a probability density $\propto a^{-1.5}$ in semi-major axis, as explained above) including both Galactic tides and stars: these differ only in terms of the set of initial conditions and the sequence of passing stars. The same three simulations have also been used as the basis of a different investigation of comet injection, which we are publishing separately in a paper submitted to Astronomy and Astrophysics.

The TAZ filling only relates to the flux of injected comets during quiescent periods, i.e., outside comet showers. Thus, for the present purpose we need to identify the latter. We consider that a star induces a shower, when its encounter parameters $d_{\odot}, V_{\star}$ and $M_{\star}$ give an estimated number of injected comets $N_{S}>25$, as computed from Eqs. (11) or (12) (depending on the stellar mass). The number 25 corresponds more or less to the background flux observed at the end of the simulations (see Fig. 8). In the case of such an encounter, we consider the shower to start at the perihelion passage of the star and to last for $10 \mathrm{Myr}$.

The full time span of each simulation is split into intervals of $100 \mathrm{Myr}$, starting $50 \mathrm{Myr}$ after the beginning. Now consider specifically the central region of the Oort cloud. For each time interval we consider the number $n_{\mathrm{TAZ}}$ of comets in the TAZ of the central region computed at the middle of the interval, and the number $n_{\mathrm{obs}}$ of injected, observable comets coming from the central region during the quiescent part of the interval. We denote the length of this quiescent part, which is obtained by subtracting all the 10 Myr shower periods, by $T_{q}$. We then compute the quiescent flux $\tau_{\text {obs }}$ 
of observable comets per Myr by:

$$
\tau_{\mathrm{obs}}=\frac{n_{\mathrm{obs}}}{T_{q}} .
$$

Let us now assume that the quiescent flux of new, observable comets per Myr is strictly proportional to the number of comets in the central TAZ, i.e.,

$$
\tau_{\mathrm{est}}=n_{\mathrm{TAZ}} \cdot C
$$

where $C$ is a constant. We will use a value of $C$ defined by the quiescent, injected comets between 300 and $500 \mathrm{Myr}$ (this period is chosen because a transient effect is still at work in our simulations during the first $200 \mathrm{Myr}^{5}$ ) (see Fouchard et al., 2005). The reason we do not choose this period of normalization even later is that we want the TAZ to be almost full, allowing more confidence in the results. We have:

$$
C=\frac{\tau_{\text {obs }}^{\star}}{n_{\text {TAZ }}^{\star}} .
$$

where the asterisks denote the specific use of the above-mentioned time interval.

Each simulation yields a different value of $C$ - namely, $C=0.644,0.569$ and 0.652 in units of $10^{-3} \mathrm{Myr}^{-1}$ - the differences being due to the specificity of each period. Because we want to treat $C$ as a constant, applicable to any simulation, we will use the mean value $C=0.622 \times 10^{-3} \mathbf{M y r}^{-1}$.

Note that, knowing the number $n_{\mathrm{CR}}$ in the central region of the Oort cloud (this is $\sim 200000$ ), $n_{\mathrm{TAZ}}$ may be replaced by $p_{\mathrm{TAZ}} p_{\mathrm{full}} n_{\mathrm{CR}}$, according to Eq. (16). Consequently, we can also compute a maximum estimated flux corresponding to a completely filled TAZ $\left(p_{\mathrm{TAZ}}=100 \%\right)$ by:

$$
\tau_{\max }=n_{\mathrm{CR}} p_{\text {full }} C
$$

For the three present sets of initial conditions, $p_{\text {full }}=2.542 \%$ for the central Oort cloud. This is slightly different from the previous value $p_{\text {full }}=2.63 \%$, since the probability density of the semi-major axes is now chosen as $\propto a_{\circ}^{-1.5}$ instead of $a_{\circ}^{-1}$. Thus, the present Oort cloud is less concentrated toward the Sun, resulting in a lower value of $p_{\text {full }}$.

Figure $8(\mathrm{a}, \mathrm{b}, \mathrm{c})$ shows in the top panels the number of injected, observable comets per interval of $20 \mathrm{Myr}$ and the number of comets in the Oort cloud at $500 \mathrm{Myr}$ intervals versus time. The largest values of $I_{\mathrm{dist}}$ for passing stars are plotted in the middle panels, and at the bottom the values of $\tau_{\text {obs }}$ for the central and entire Oort cloud are plotted along with $\tau_{\text {est }}$ and $\tau_{\max }$. Each figure shows the output of one of the three simulations (Fig. 8a corresponds to the model C(all) already used in Sect. 4.2).

\footnotetext{
${ }^{5}$ This transient effect is due to the flat initial distribution of arguments of perihelia, which is not stable under the action of the Galactic tides. The tides modify this distribution, resulting in an increase of the flux at the beginning of the integrations.
} 
The agreement between $\tau_{\text {est }}$ and $\tau_{\text {obs }}$ for the central Oort cloud is rather good and improves with time along the span of the simulations. Thus the assumption behind the calculation of $\tau_{\text {est }}$ appears warranted. This highlights the importance of the Galactic tides for comet injection from the central Oort cloud. The fluctuations shown by $\tau_{\text {obs }}$ are partly due to small number statistics, since $T_{q}$ is sometimes rather small, and partly to inherent fluctuations in the efficiency of non-shower making stars between different intervals. The relatively small difference between $\tau_{\text {obs }}$ computed for the central and entire Oort cloud during the later part of the simulations shows that the central Oort cloud gives the major contribution to the quiescent flux of observable comets.

In addition, considering $\tau_{\max }$ as an indicator of the number of comets in the central Oort cloud, we observe that the overall depletion of the Oort cloud comes mainly from its central part, whose population is depleted by almost a factor two in each simulation.

It is difficult to disentangle the role of individual stars in these simulations.For each sufficiently large increase of $\tau_{\text {est }}$ as judged by visual inspection of Fig. 8, Table 4 gives: the period during which the increase occurs (column 1), the TAZ filling expressed by the percentages $p_{\text {TAZ }}^{(i)}$ and $p_{\text {TAZ }}^{(f)}$ at, respectively, the beginning $(i)$ and the end $(f)$ of the $100 \mathrm{Myr}$ periods (column 2), and the mass(es) of the $\operatorname{star}(\mathrm{s})$ with the largest value(s) of $I_{\text {dist }}$ (if a single star is clearly identified, only one mass is given, while otherwise several masses are listed) (column 3). This Table summarizes the most significant cases of instant TAZ filling, leading to "comet drizzles" as discussed in Sect. 3.5.

Most of the guilty stars are high-mass stars $\left(M_{\star}>1.2 M_{\odot}\right)$. Among the ten comet drizzles listed, five involve stars of lower masses, but one of these is a marginal case $\left(M_{\star}=1.1 M_{\odot}\right)$, and two others involve high-mass stars as well. A striking feature in Fig. 8 is the frequent occurrence of large $I_{\text {dist }}$ values for the lightest M5 stars. This is due to the large overall frequency of these encounters, but note that Fig. 5 showed that even large $I_{\text {dist }}$ values mostly lead to poor TAZ filling for those stars.

As seen in Fig. 8, the comet drizzles are generally associated to one comet shower (except for the one starting between 0.5 and $0.6 \mathrm{Gyr}$ in the first simulation, where three showers seem to contribute to the TAZ filling). On the other hand, showers do not always give rise to visible drizzles, since it is harder to detect a TAZ filling than a loss cone filling. This is mainly due to the fact that the TAZ is never empty, whereas the loss cone is generally empty - at least for moderate semi-major axes.

The influence of these few stellar encounters on the long term flux is evident. Indeed, from a statistical 


\begin{tabular}{|c|c|c|}
\hline Period (Gyr) & $p_{\mathrm{TAZ}}^{(i)}-p_{\mathrm{TAZ}}^{(f)}(\%)$ & $M_{\star}\left(M_{\odot}\right)$ \\
\hline \multicolumn{3}{|c|}{ Simulation \# 1} \\
\hline $0.5-0.6$ & $83-89$ & $4,0.21,4$ \\
\hline $1.8-1.9$ & $71-83$ & 2.1 \\
\hline $3.1-3.2$ & $62-69$ & $4,0.21$ \\
\hline \multicolumn{3}{|c|}{ Simulation \# 2} \\
\hline $1.8-1.9$ & $70-82$ & 1.3 \\
\hline $4.1-4.2$ & $59-70$ & 0.78 \\
\hline \multicolumn{3}{|c|}{ Simulation \# 3} \\
\hline $1.4-1.5$ & $68-78$ & 2.1 \\
\hline $2.7-2.8$ & $78-83$ & 1.3 \\
\hline $3.5-3.6$ & $74-86$ & 0.21 \\
\hline $4.1-4.2$ & $75-88$ & 9 \\
\hline $4.8-4.9$ & $76-101$ & 1.1 \\
\hline
\end{tabular}

Table 4: The periods during which clear increases of $\tau_{\text {est }}$ are observed in Fig. 8, the values of the TAZ filling at the beginning $\left(p_{\mathrm{TAZ}}^{(i)}\right)$ and at the end $\left(p_{\mathrm{TAZ}}^{(f)}\right)$, and the masses of the stars with the largest values of $I_{\mathrm{dist}}$ that passed during the respective periods.

point of view, the three sets of stellar encounters should lead to an identical background flux, whereas the comet showers and the comet drizzles may differ. In Fig. 8 we see that this is verified.

We observe in the top plots of Fig. 8(a,b,c) that during the last Gyr the first simulation has no strong stellar encounters, whereas the third one is affected by two such encounters yielding two comet drizzles (Table 4). The final observed fluxes from the central Oort cloud are $0.52 \mathrm{Myr}^{-1}$ for the first simulation, and $0.97 \mathrm{Myr}^{-1}$ for the third one. Thus the third simulation produces 1.87 times more comets from the central cloud at the end than the first one does.

This, again, is directly related to the TAZ filling, since at the end the central TAZ is filled only to $55 \%$ in the first simulation, whereas for the third simulation it is almost completely filled (97\%). Considering the whole Oort cloud, the third simulation produces 1.39 times more observable comets at the end than the first one does. This ratio is lower, since in our dynamical model the contribution from the inner cloud outside showers is negligible, and the contribution from the outer cloud is not affected by the comet drizzles - its TAZ being always full.

Consequently, it appears that the flux of comets toward the observable region is closely related to the population of comets inside the TAZ. At the end of the simulations, we note that a TAZ filled at the level of $50-60 \%$ is guaranteed by the "background" passing stars. However, a single passing star - preferably massive - is able to fill the TAZ completely, resulting in an almost $100 \%$ increase of the flux of observable comets from the central cloud and of $40 \%$ from the entire cloud. Such increases may last for more than 100 Myr. 


\section{Conclusions}

We have investigated the effect of stellar perturbations on the flux of comets coming from the Oort cloud into the observable region. We considered 13 different spectral types including 11 main sequence types, white dwarfs and giant stars.

As a first result it has been demonstrated and verified quantitatively (to our knowledge the first time) that high-mass stars $\left(M_{\star}>2 M_{\odot}\right)$ - and in particular the most massive ones like the B0 stars - inject comets from large parts of the cloud during each encounter, whereas light stars $\left(M_{\star}<1 M_{\odot}\right)$ mainly affect comets in their close vicinity. The number of injected comets is generally much larger for high-mass than for low-mass stars, thus partly compensating for the relative rarity of their encounters. Moreover, the efficiency of the stars to fill the Tidally Active Zone (the region from which the Galactic tides are able to decrease the perihelion distance of a comet below $5 \mathrm{AU}$ ) is also strongly dependent on the stellar mass.

Let us compare our study with that of Heisler et al. (1987). They simulated the cloud for only about $300 \mathrm{Myr}$ and were thus not able to see the effects of very massive stars in creating comet showers. Their conclusion was that such stars are too rare to be efficient, but ours is quite different, since we follow the cloud for the full age of the Solar System. We may add that even more massive perturbers, like the GMCs, have so far been neglected in all Oort cloud simulations of the present type. Only analytic studies have been devoted to their effect on the Oort cloud (Hut and Tremaine, 1985) or wide binaries (Weinberg et al. , 1987).

We have shown that the TAZ filling due to stellar encounters is a fundamental condition for injection of comets into the observable region. Indeed, as was already asserted in RFFV08, we have shown that the filling of the TAZ is directly related both to the synergy between the Galactic tides and stellar perturbations and to the flux of injected, observable comets.

The massive stars not only induce stronger comet showers than light stars do, but they are also able to trigger a synergy with the Galactic tides that may last for a few hundred Myr. Because of this synergy, a single star - preferably massive - is able to almost double the flux of comets from the central cloud for more than $100 \mathrm{Myr}$. If one considers the entire Oort cloud, the flux of comets is thus multiplied by about 1.4. We have called such events comet drizzles. It is important to keep in mind that the current flux of new Oort cloud comets may be influenced by an encounter with a massive star that occurred more than $100 \mathrm{Myr}$ ago.

In conclusion, one may consider the flux of observable comets as a two steps mechanism: (i) First the comet is injected into the TAZ under the effects of stellar perturbations. This zone acts like a "waiting room" before entering the loss cone. (ii) Then the comet is sent from the TAZ into the depth of the loss 
cone under the action of the tides. Consequently, the long term synergy between tides and passing stars is crucial in order to maintain a flux of observable comets on a long time span. This is yet another new feature as compared with Heisler et al. (1987). Their 300 Myr simulations started with a thermalized cloud, i.e., a filled TAZ, and there was no time to empty it. Thus they found that comet injection is dominated by the Galactic tide. We too find that this is the case at the present time, but we emphasize that the reason is the long-term refilling of the TAZ by stellar encounters, especially those of high-mass stars.

As a final remark, note that on a time scale of $\sim 100$ Myr, the Sun is orbiting the Galactic center and oscillating "vertically" through the Galactic mid-plane. Thus, the frequency of stellar encounters - and in particular that of high-mass stars - may show important variations, and it will eventually be important to include those variations (Gardner et al., 2010) when studying the likely origin of the currently observed new comets. While there is currently no way to verify observationally, whether we are living in a comet drizzle, the results of Gardner et al. (2010) show that the current location of the Sun near the Galactic mid-plane and near its peri-Galacticon causes the present, tidally induced flux of new comets to be higher (likely by $\sim 35 \%$ ) than the long-term average. As far as we can tell, the amplitudes of both variations are similar.

\section{Acknowledgements}

We thank Ramon Brasser and Nathan Kaib, who as referees made numerous suggestions that greatly helped to improve the quality of the paper. This work was made possible due to a research Grant from the Polish Government (NN 203392 734). In addition, HR was supported by a Swedish National Space Board Grant (119/07), and the work of GBV was supported by the Italian Space Agency, under contract ASI/INAF I/015/07/0.

\section{References}

M. E. Bailey, C. R. Stagg, Cratering constraints on the inner Oort cloud - Steady-state models, MNRAS 235 (1988) 1-32. S. Breiter, P. Dybczyński, A. Elipe, The action of the Galactic disk on the Oort cloud comets, A\&A 315 (1996) 618-624.

S. Breiter, M. Fouchard, R. Ratajczak, W. Borczyk, Two fast integrators for the Galactic tide effects in the Oort Cloud, MNRAS 377 (2007) 1151-1162.

J. Byl, Galactic perturbations on nearly-parabolic cometary orbits, Moon and Planets 29 (1983) 121-137.

M. Duncan, T. Quinn, S. Tremaine, The formation and extent of the solar system comet cloud, AJ 94 (1987) 1330-1338.

P. A. Dybczyński, Simulating observable comets. I. The effects of a single stellar passage through or near the Oort cometary cloud, AAP396(2002) 283-292.

P. A. Dybczyński, Impulse approximation improved, Celestial Mechanics and Dynamical Astronomy58(1994) 139-150.

M. Fouchard, Ch. Froeschlé, J. J. Matese, G. Valsecchi, Comparison between Different Models of Galactic Tidal Effects on Cometary Orbits, Celestial Mechanics and Dynamical Astronomy 93 (2005) 229-262.

M. Fouchard, Ch. Froeschlé, S. Breiter, R. Ratajczak, H. Valsecchi, G.B. Rickman, Methods to Study the Dynamics of the Oort Cloud Comets II: Modelling the Galactic Tide, in: D. Benest, Cl. Froeschlé, E. Lega (Eds.), Topics in Gravitational dynamics, vol. 729 of Lecture Notes in Physics, Berlin Springer Verlag, in press, 271-293, 2007. 
M. Fouchard, Ch. Froeschlé, H. Rickman, G. B. Valsecchi, Dynamical Features of the Oort Cloud Comets, in: J. Souchay \& R. Dvorak (Ed.), Lecture Notes in Physics, Berlin Springer Verlag, vol. 790 of Lecture Notes in Physics, Berlin Springer Verlag, 2010.

J. García-Sánchez, P. R. Weissman, R. A. Preston, D. L. Jones, J.-F. Lestrade, D. W. Latham, R. P. Stefanik, J. M. Paredes, Stellar encounters with the solar system, AAP379(2001)634-659.

E. Gardner, P. Nurmi, C. Flynn, S. Mikkola, The effect of the Solar motion on the flux of long-period comets,MNRAS411(2011)947-954

J. Heisler, S. Tremaine, The influence of the galactic tidal field on the Oort comet cloud, Icarus 65 (1986) 13-26.

J. Heisler, S. Tremaine, C. Alcock, The frequency and intensity of comets showers from the Oort cloud, Icarus 70 (1987) 269-288.

J. Holmberg, C. Flynn, The local density of matter mapped by Hipparcos, MNRAS313(2000)209-216.

P. Hut, S. Tremaine, Have interstellar clouds disrupted the Oort comet cloud?, AJ 90(1985) 1548-1557.

N. A. Kaib, T. Quinn, Reassessing the Source of Long-Period Comets, Science 325(2009) 1234-

A. J. Maciejewski, H. Prȩtka, Galactic disc tidal action and observability of the Oort cloud comets, AAP336(1998) $1065-1071$.

J. J. Matese, J. J. Lissauer, Characteristics and Frequency of Weak Stellar Impulses of the Oort Cloud, Icarus 157 (2002) $228-240$.

J. J. Matese, P. G. Whitman, A model of the galactic tidal interaction with the Oort comet cloud, Celest. Mech. Dynam. Astron. 54 (1992) 13-35.

J. H. Oort, The structure of the cloud of comets surrounding the Solar System and a hypothesis concerning its origin, Bull. Astron. Inst. Neth. 11 (1950) 91-110.

H. Rickman, Stellar perturbations of orbits of long-period comets and their significance for cometary capture, Bulletin of the Astronomical Institutes of Czechoslovakia 27 (1976) 92-105.

H. Rickman, M. Fouchard, G. B. Valsecchi, Ch. Froeschlé, Algorithms for Stellar Perturbation Computations on Oort Cloud Comets, Earth Moon and Planets 97 (2005) 411-434.

H. Rickman, M. Fouchard, Ch. Froeschlé, G. B. Valsecchi, Injection of Oort Cloud Comets: The Fundamental Role of Stellar Perturbations, Celestial Mechanics and Dynamical Astronomy 102 (2008) 111-132.

H. Rickman, Ch. Froeschlé, Cl. Froeschlé, G. B. Valsecchi, Stellar perturbations on the scattered disk, AAP 428 (2004) 673-681.

M. D. Weinberg, S. L. Shapiro, I. Wasserman, The dynamical fate of wide binaries in the solar neighborhood, APJ 312 (1987) $367-389$.

P. Wiegert, S. Tremaine, The Evolution of Long-Period Comets, Icarus 137 (1999) 84-121. 
(a)

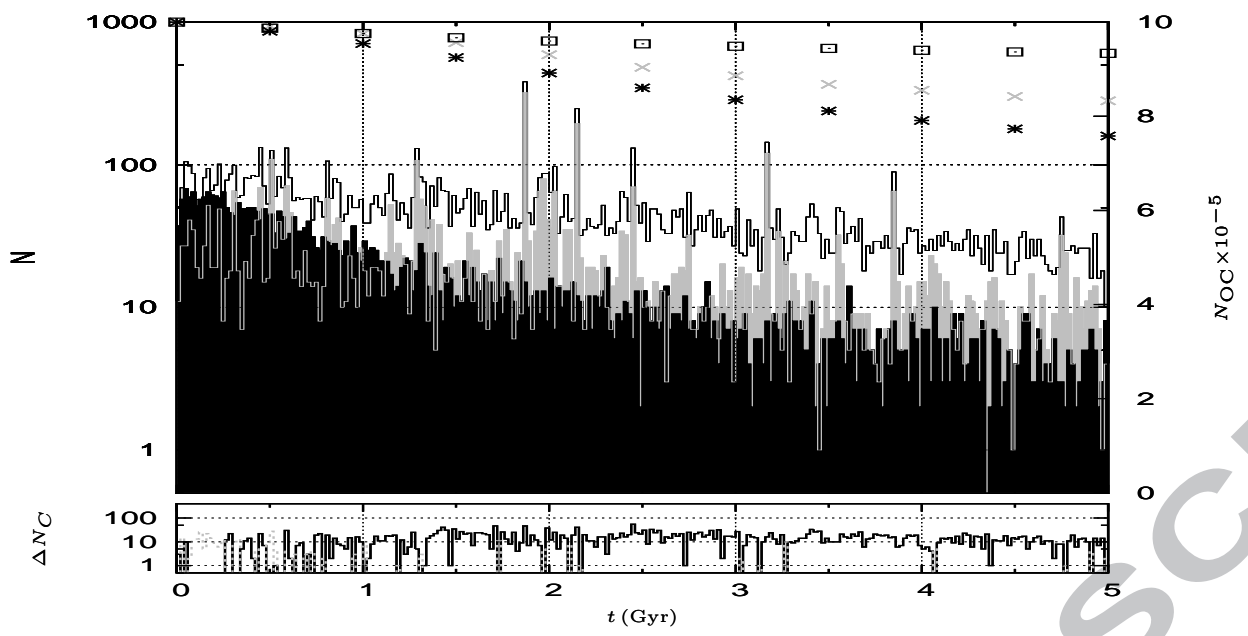

(b)

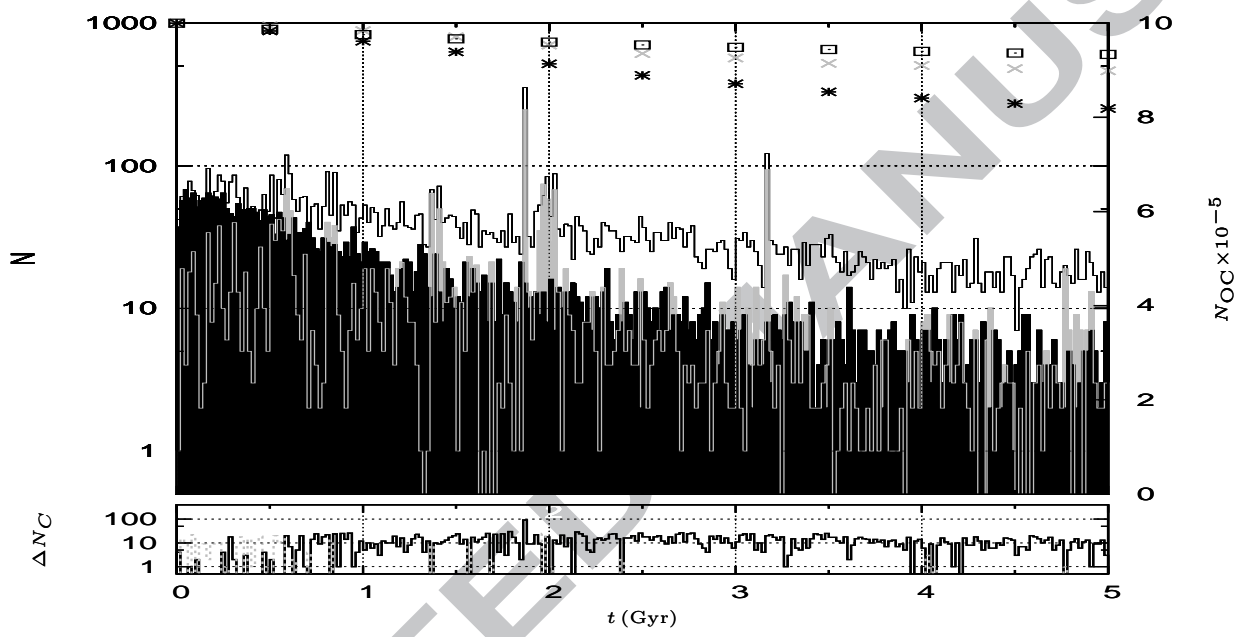

(c)

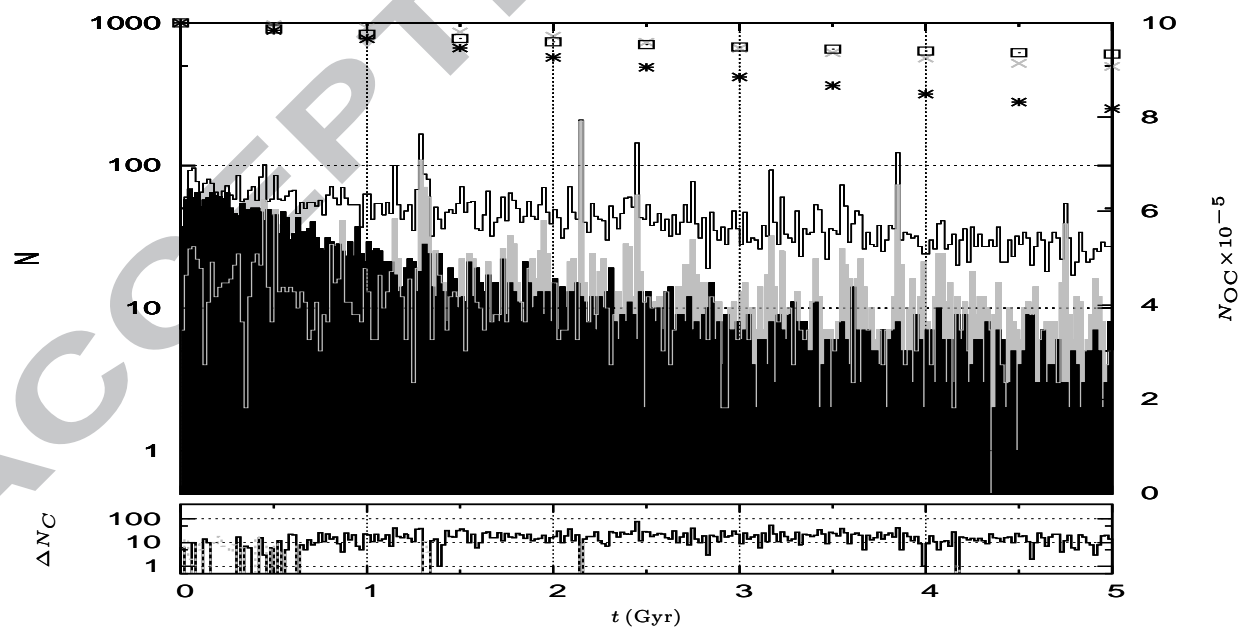

Figure 7: The upper diagrams show the number of injected comets per $20 \mathrm{Myr}$ versus time (in Gyr). The black histograms correspond to the tide-only model $\left(N_{G}\right)$, the grey ones to the star-only model $\left(N_{S}\right)$, and the white ones to the combined model $\left(N_{C}\right)$. The lower graphs exhibit the values of $\Delta N_{C}=N_{C}-N_{G}-N_{S}$ in each 20 Myr time bin. When positive, $\Delta N_{C}$ is plotted by a full black line; otherwise we use a dotted grey line. Figure $(a)$ is for the all-stars models, $(b)$ for heavy-stars models and (c) for light-stars models. The black asterisks, grey asterisks and black rectangles in the upper panels indicate the number of comets remaining in the Oort cloud at intervals of 0.5 Gyr scale on the right axis for the combined models, the only stars models and only tides models respectively. 
(a)
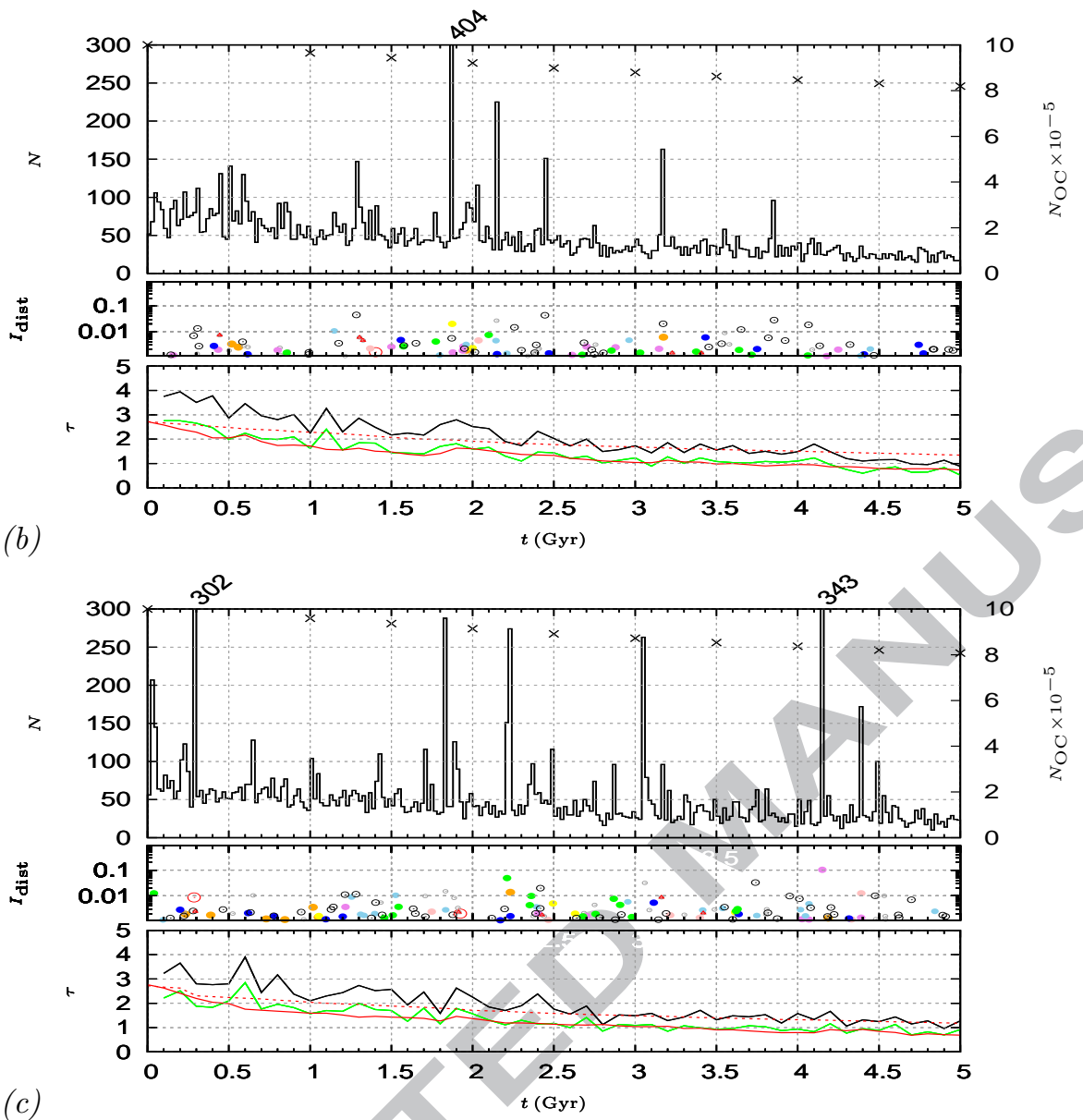

(c)

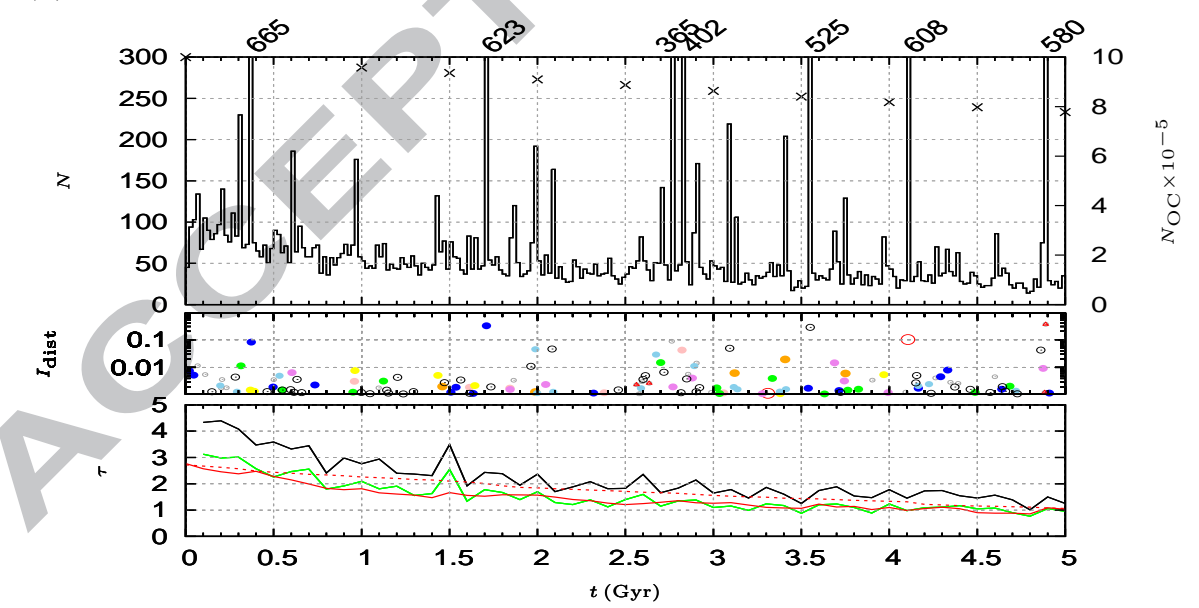

Figure 8: In each diagram (a,b,c) the top panel gives the number of injected comets per 20 Myr (histogram) and the number of comets in the Oort cloud every $500 \mathrm{Myr}$ (crosses). The middle panel shows the largest values of $I_{\text {dist }}$ for passing stars (the colours denote different stellar types as in previous Figures). The bottom panel shows the flux of injected, observable comets per Myr outside showers during each 100 Myr interval $\left(\tau_{\text {obs }}\right)$ for the central Oort cloud (green line) and the entire Oort cloud (black line), the estimated flux $\left(\tau_{\text {est }}\right)$ for the central Oort cloud (full red line) and the flux $\left(\tau_{\max }\right)$ considering a filled TAZ (dashed red line). The figures represent three simulations using different sets of initial conditions and sequences of passing stars. 
$>$ The effects of passing stars on the flux of observable comets is studied.

$>$ Massive stars inject observable comets much more efficiently than light stars do.

$>$ The long term synergy between stars and Galactic tide is explained.

$>$ Stars send comets into orbits from which the tides may make them observable.

$>$ Single massive stars may increase the flux significantly for more than $100 \mathrm{Myr}$. 